\title{
Dynamic Reconfiguration of Visuomotor-Related Functional Connectivity Networks
}

\author{
(1DAndrea Brovelli, ${ }_{1}^{1}$ - Jean-Michel Badier, ${ }^{2,3}$ Francesca Bonini, ${ }^{2,3}$ Fabrice Bartolomei, ${ }^{2,3}$ @olivier Coulon, ${ }^{1,4 *}$ \\ and (Duillaume Auzias ${ }^{1,4 *}$ \\ ${ }^{1}$ Institut de Neurosciences de la Timone, Unité Mixte de Recherche 7289, Aix Marseille Université, Centre National de la Recherche Scientifique, 13385 \\ Marseille, France; ${ }^{2}$ Aix Marseille Université, Institut de Neurosciences des Systèmes Unité Mixte de Recherche Scientifique 1106, 13005 Marseille, France; \\ ${ }^{3}$ Inserm, Unité Mixte de Recherche Scientifique 1106, 13005 Marseille, France; and ${ }^{4}$ Aix-Marseille Université, Centre National de la Recherche Scientifique, \\ Laboratoire des Sciences de l'Information et des Systèmes Unité Mixte de Recherche 7296 Marseille, France
}

Cognitive functions arise from the coordination of large-scale brain networks. However, the principles governing interareal functional connectivity dynamics (FCD) remain elusive. Here, we tested the hypothesis that human executive functions arise from the dynamic interplay of multiple networks. To do so, we investigated FCD mediating a key executing function, known as arbitrary visuomotor mapping, using brain connectivity analyses of high-gamma activity recorded using MEG and intracranial EEG. Visuomotor mapping was found to arise from the dynamic interplay of three partly overlapping cortico-cortical and cortico-subcortical functional connectivity (FC) networks. First, visual and parietal regions coordinated with sensorimotor and premotor areas. Second, the dorsal frontoparietal circuit together with the sensorimotor and associative frontostriatal networks took the lead. Finally, cortico-cortical interhemispheric coordination among bilateral sensorimotor regions coupled with the left frontoparietal network and visual areas. We suggest that these networks reflect the processing of visual information, the emergence of visuomotor plans, and the processing of somatosensory reafference or action's outcomes, respectively. We thus demonstrated that visuomotor integration resides in the dynamic reconfiguration of multiple cortico-cortical and cortico-subcortical FC networks. More generally, we showed that visuomotor-related FC is nonstationary and displays switching dynamics and areal flexibility over timescales relevant for task performance. In addition, visuomotor-related FC is characterized by sparse connectivity with density $<10 \%$. To conclude, our results elucidate the relation between dynamic network reconfiguration and executive functions over short timescales and provide a candidate entry point toward a better understanding of cognitive architectures.

Key words: dynamic reconfiguration; executive functions; functional connectivity dynamics; high-gamma activity; MEG; SEEG

Significance Statement

Executive functions are supported by the dynamic coordination of neural activity over large-scale networks. The properties of large-scale brain coordination processes, however, remain unclear. Using tools combining MEG and intracranial EEG with brain connectivity analyses, we provide evidence that visuomotor behaviors, a hallmark of executive functions, are mediated by the interplay of multiple and spatially overlapping subnetworks. These subnetworks span visuomotor-related areas, the corticocortical and cortico-subcortical interactions of which evolve rapidly and reconfigure over timescales relevant for behavior. Visuomotor-related functional connectivity dynamics are characterized by sparse connections, nonstationarity, switching dynamics, and areal flexibility. We suggest that these properties represent key aspects of large-scale functional networks and cognitive architectures.

\section{Introduction}

The dynamic coordination of neural activity over large-scale networks is thought to support cognitive functions (Varela et al., 2001; Bressler and Menon, 2010; von der Malsburg et al., 2010).
Growing evidence from fMRI has shown that spontaneous and task-related activity is composed of multiple and spatially overlapping subnetworks that dynamically evolve over tens of seconds to minutes (Hutchison et al., 2013; Yeo et al., 2014; Allen et 
al., 2014; Calhoun et al., 2014; Cole et al., 2014; Zalesky et al., 2014; Hansen et al., 2015). Indeed, dynamic network reconfiguration has been suggested to represent a fundamental neurophysiological process supporting executive function (Bassett et al., 2011; Braun et al., 2015).

A hallmark of executive function is the ability to rapidly associate arbitrary actions to visual inputs and internal goals. This ability, known as arbitrary visuomotor mapping, recruits a large-scale network comprising the sensorimotor and frontoparietal circuits, in addition to medial prefrontal areas and basal ganglia (Wise et al., 1996; Murray et al., 2000; Passingham et al., 2000; Wise and Murray, 2000; Hadj-Bouziane et al., 2003; Petrides, 2005). In the current study, the goal was to investigate whether visuomotor mapping results from the dynamic interplay of multiple subnetworks. More precisely, we tested the hypothesis that multiple subnetworks reconfigure in a dynamic fashion over timescales relevant for executive behaviors.

To do so, we exploited the highgamma activity (HGA; ranging from $\sim 60$ to $150 \mathrm{~Hz}$ ), which reflects populationlevel local neural activity (Ray et al., 2008; Ray and Maunsell, 2011). In humans, power modulations in the HGA range are commonly recorded using MEG and intracranial EEG to map task-related brain regions (Brovelli et al., 2005; Crone et al., 2006; Vidal et al., 2006; Ball et al., 2008; Jerbi et al., 2009; Darvas et al., 2010; Lachaux et al., 2012; Cheyne and Ferrari, 2013; Ko et al., 2013). In addition, highgamma-power modulations can be used to characterize functional connectivity (FC) among brain regions supporting executive functions (Brovelli et al., 2015).

Here, we predicted visuomotor mapping to reflect an initial activation of visual circuits mediating the processing of sensory input, followed by frontoparietal and motor networks for visuomotor integration and motor planning. We tested these hypotheses in a group of healthy participants during MEG recordings by combining FC analysis of atlas-based HGA with methods from network science and graph theory. In particular, we investigated the presence of multiple visuomotor-related spatiotemporal patterns through the analysis of FC dynamics (FCD). Intracranial assessment of HGA activations from MEG was also performed in

(Grant ANR-11-INBS-0006). We thank Faical Isbaine and Sophie Chen for help with the MEG experiments and Patrick Marquis and Catherine Liegeois-Chauvel for help with the SEEG experiments.

The authors declare no competing financial interests.

*0.C. and G.A. contributed equally to this work.

Correspondence should be addressed to Andrea Brovelli, Institut de Neurosciences de la Timone (INT), UMR 7289

CNRS, Aix Marseille University, Campus de Santé Timone, 27 Bd. Jean Moulin, 13385 Marseille, France. E-mail: andrea.brovelli@univ-amu.fr.

DOI:10.1523/JNEUROSCI.1672-16.2016

Copyright $\odot 2017$ the authors $\quad 0270-6474 / 17 / 370840-15 \$ 15.00 / 0$

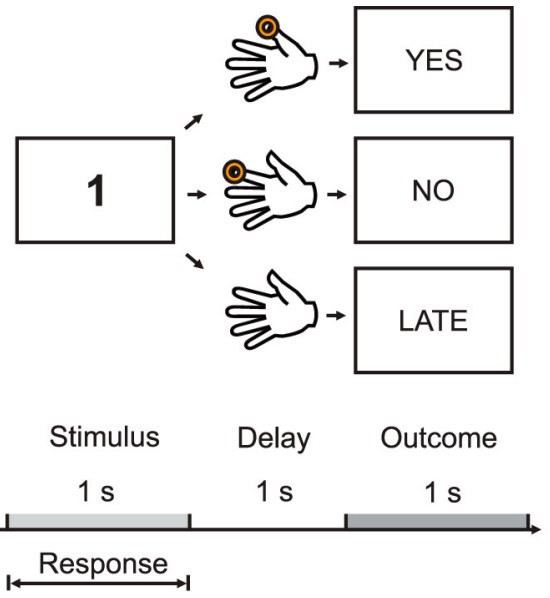

\begin{tabular}{|c|c|c|c|}
\hline Baseline & Stimulus & Delay & Outcome \\
\hline $2-3 s$ & $1 \mathrm{~s}$ & $1 \mathrm{~s}$ & $1 \mathrm{~s}$ \\
\hline
\end{tabular}
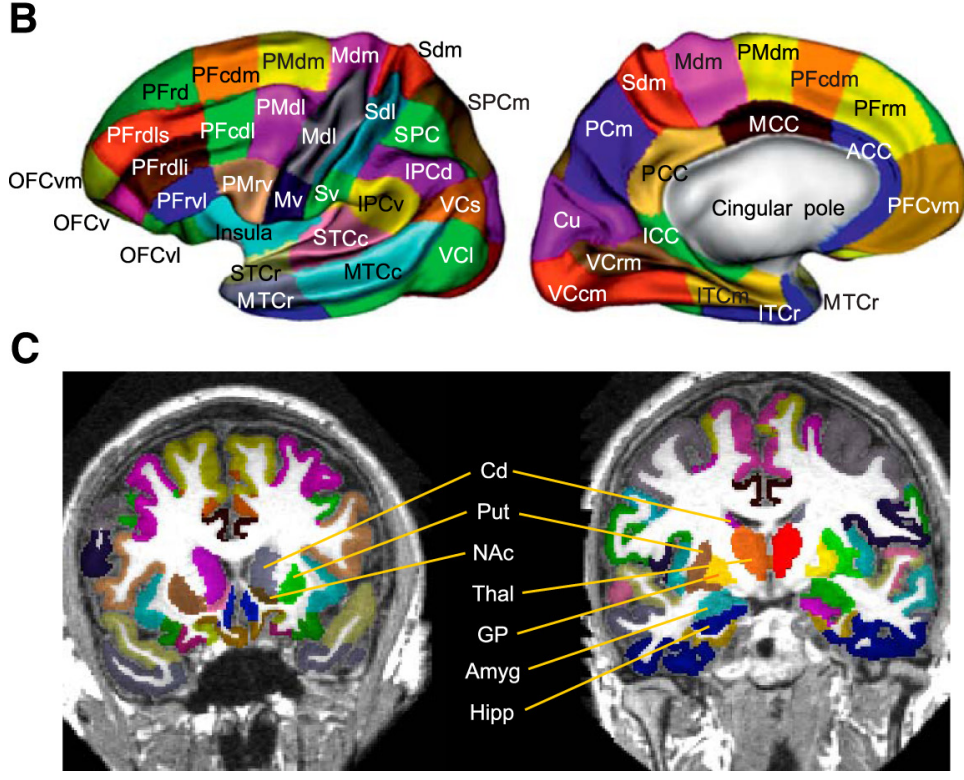

Figure 1. A, Arbitrary visuomotor mapping task. B, MarsAtlas: cortical parcellation displaying the anatomical gradients both in the rostrocaudal and dorsoventral directions; for a detailed description, see Auzias et al. (2016). C, MarsAtlas: single-subject exemplar volumetric representation displaying subcortical regions included in the atlas: nucleus accumbens, amygdala, hippocampus, globus pallidus, putamen, caudate nucleus, and thalamus.

three epileptic patients performing the same task during stereoEEG (SEEG) recordings.

\section{Materials and Methods}

Experimental procedure and data acquisition

Experimental conditions and behavioral tasks. Eleven healthy participants and three epileptic patients accepted to take part in our study. Healthy participants were right handed and the average age was $\sim 23$ years $(\mathrm{SD}=$ 3.8 years); 4 were females and 7 males. All gave written informed consent according to established institutional guidelines and local ethics committee, and received monetary compensation ( $€ 50$ ). Three patients (one right-handed female age 29, two right-handed males age 29 and 43) undergoing presurgical evaluation of drug-resistant epilepsy (Epilepsy Unit, La Timone Hospital, Marseille, France) participated in this study. They all gave their informed consent before their participation. The SEEG study was approved by the institutional review board of the French Institute of Health. Healthy participants and patients performed the same behavioral task. Participants were asked to perform an associative visuomotor mapping task in which the relation between visual stimulus and motor response is arbitrary and deterministic (Wise and Murray, 2000; Brovelli et al., 2015). As shown in Figure $1 A$, the task required 
participants to perform a finger movement associated to a digit number: digit " 1 " instructed the execution of the thumb, " 2 " for the index finger, " 3 " for the middle finger, and so on. Maximal reaction time was $1 \mathrm{~s}$. After a fixed delay of $1 \mathrm{~s}$ after the disappearance of the digit number, an outcome image was presented for $1 \mathrm{~s}$ and informed the subject whether the response was correct, incorrect, or too late (if the reaction time exceeded $1 \mathrm{~s})$. Incorrect and late trials were excluded from the analysis because they were either absent or very rare (i.e., maximum two late trials per session). The next trial started after a variable delay ranging from 2 to $3 \mathrm{~s}$ (randomly drawn from a uniform distribution) with the presentation of another visual stimulus. Each participant performed two sessions of 60 trials each (total of 120 trials). Each session included three digits randomly presented in blocks of three trials. The average reaction time was $0.504 \pm 0.004 \mathrm{~s}$ (mean \pm SEM).

Anatomical, functional, and behavioral data acquisition in healthy participants. Anatomical MRI images were acquired for healthy participant using a $3 \mathrm{~T}$ whole-body imager equipped with a circular polarized head coil. High-resolution structural T1-weighted anatomical image (inversion-recovery sequence, $1 \times 0.75 \times 1.22 \mathrm{~mm}$ ) parallel to the anterior commissure-posterior commissure plane, covering the whole brain, were acquired. MEG recordings were performed using a 248 magnetometers system (4D Neuroimaging Magnes 3600). Visual stimuli were projected using a video projection and motor responses were acquired using a LUMItouch optical response keypad with five keys. Presentation software was used for stimulus delivery and experimental control during MEG acquisition. Reaction times were computed as the time difference between stimulus onset and motor response. Sampling rate was 2034.5 $\mathrm{Hz}$. Location of the participant's head with respect to the MEG sensors was recorded both at the beginning and end of each session to exclude sessions and/or participants with large head movements. For each session and participant, the displacement between the beginning and end of a session was computed. A supine position was chosen to minimize head movements. This cutoff was decided by considering the spatial distance between sources ( $5 \mathrm{~mm}$ ), as described in the following sections. None of the participants moved $>3 \mathrm{~mm}$ during all sessions. Therefore, all participants were considered for further analysis.

Anatomical, functional, and behavioral data acquisition in epileptic patients. The surgical treatment of drug-resistant epilepsy may require direct intracerebral recording of cortical activity IEEG in multiple brain areas to localize the epileptic tissue to be removed. Before SEEG, all patients had high-resolution MRI performed with a $3 \mathrm{~T}$ Siemens Magnetom scanner, including a 3D T1-weighted acquisition. Intracerebral multiple contacts electrodes (10-15 contacts, length: $2 \mathrm{~mm}$, diameter: $0.8 \mathrm{~mm}, 1.5 \mathrm{~mm}$ apart) were implanted using a stereotactic method. A postoperative computerized tomography (CT) scan without contrast was used to verify the absence of bleeding and the location of each recording lead. During this presurgical evaluation period, patients were asked to participate in our behavioral protocol at the Timone Hospital. They were seated in a Faraday cage and stimuli were presented on a display monitor at $70 \mathrm{~cm}$ to patient's eyes with an angular size of $1.26^{\circ}$. Presentation software was used for stimulus delivery and experimental control during SEEG acquisition. Motor responses were acquired using a five-button response pad. SEEG signals were acquired on referential montage with a sampling frequency of $1000 \mathrm{~Hz}$ and an acquisition bandpass filter between 0.1 and $200 \mathrm{~Hz}$.

\section{Brain parcellation}

To map brain activations and FC patterns to specific anatomical brain networks, single-subject brain parcellation can be created from macroanatomical information, such as primary and secondary sulci using either volume-based (Lancaster et al., 2000; Tzourio-Mazoyer et al., 2002) or surface-based (Van Essen and Drury, 1997; Fischl et al., 2002; Desikan et al., 2006) algorithms. Recent developments now allow single-subject cortical parcellation complying with a model of anatomofunctional gradients in the rostrocaudal and dorsoventral directions (Auzias et al., 2013) optimized for functional mapping using HGA (Auzias et al., 2016). Such an approach allows group-level analyses and comparison between individual patients and healthy participants in control group. We created a whole-brain parcellation including cortical (Fig. 1B) and subcortical
(Fig. 1C) regions based on macro-anatomical information. The identification of the cortical regions requires several processing steps. After denoising using a nonlocal means approach (Coupé et al., 2008), T1weighted MR-images were segmented using the FreeSurfer "recon-all" pipeline (http://freesurfer.net). Gray and white matter segmentations of each hemisphere were imported into the BrainVisa software and processed using the Morphologist pipeline procedure (http://brainvisa.info). White matter and pial surfaces were reconstructed and triangulated and all sulci were detected and labeled automatically (Mangin et al., 2004; Perrot et al., 2011). A parameterization of each hemisphere white matter mesh was performed using the Cortical Surface Toolbox (http://brainvisa.info/web/cortical_surface.html). This resulted in a 2D orthogonal system defined on the white matter mesh constrained by a set of primary and secondary sulci (Auzias et al., 2013). This parameterization naturally leads to a complete parcellation of the cortical surface, the MarsAtlas model (Auzias et al., 2016).

MarsAtlas complies with the dorsoventral and rostrocaudal trends of cortical organization (Pandya and Yeterian, 1985; Régis et al., 2005) and provides a good level of both functional segregation and intersubject matching for functional analysis using single-trial MEG HGA (Auzias et al., 2016). The resulting cortical surface parcellation was then propagated to the volume-based gray matter segmentation using a front propagation from the surface through the volumetric cortex segmentation (Cachia et al., 2003), thus producing a volume-based parcellation of the entire cortex. The parcels corresponding to the subcortical structures were extracted using Freesurfer (Fischl et al., 2002). The subcortical structures included in the brain parcellation were the caudate nucleus, putamen, nucleus accumbens, globus pallidus, thalamus, amygdala, and hippocampus. The whole-brain parcellation therefore comprised 96 areas (41 cortical and 7 subcortical areas per hemisphere; Fig. 1). All of these processing steps can be performed using the BrainVisa neuroimaging platform (http://brainvisa.info/web/index.html). MarsAtlas is included in the cortical surface toolbox.

\section{Single-trial HGA in MarsAtlas}

Preprocessing and spectral analysis of MEG and SEEG signals. The preprocessing and spectral analyses steps for MEG and SEEG signals were identical. Concerning SEEG signals, an electrode's contacts in the epileptogenic zone were excluded from the analysis. SEEG contacts outside of the epileptogenic zone were chosen for analysis. In addition, epochs with signs of epileptic activity were removed. MEG and SEEG signals were first down-sampled to $1 \mathrm{kHz}$, low-pass filtered to $250 \mathrm{~Hz}$, and then segmented into epochs aligned on finger movement (i.e., button press). Epoch segmentation was also performed on stimulus onset and the data from -0.5 and -0.1 s before stimulus presentation were taken as baseline activity for the calculation of the single-trial HGA. Artifact rejection was performed semiautomatically and by visual inspection. For each movement-aligned epoch and channel, the signal variance and $z$-value were computed over time and taken as relevant metrics for the identification of artifact epochs. All trials with a variance $>1.5^{\star} 10-24$ across channels were excluded from further search of artifacts. Metrics such as the $z$-score, absolute $z$-score, and range between the minimum and maximum values were also inspected to detect artifacts. Channels and trials displaying outliers were removed. Two MEG sensors were excluded from the analysis for all subjects.

Spectral density estimation was performed using multitaper method based on discrete prolate spheroidal (slepian) sequences (Percival and Walden, 1993; Mitra and Pesaran, 1999). To extract HGA from 60 to 120, MEG time series were multiplied by $k$ orthogonal tapers $(k=8)(0.15 \mathrm{~s}$ in duration and $60 \mathrm{~Hz}$ of frequency resolution, each stepped every $0.005 \mathrm{~s}$ ), centered at $90 \mathrm{~Hz}$ and Fourier-transformed. Complex-valued estimates of spectral measures, $X_{\text {sensor }}^{n}(t, k)$, including cross-spectral density matrices, were computed at the sensor level for each trial $n$, time $t$, and taper $k$.

$M E G$ source analysis and HGA. Source analysis requires a physical forward model or lead field, which describes the electromagnetic relation between sources and MEG sensors. The lead field combines the geometrical relation of sources (dipoles) and sensors with a model of the conductive medium (i.e., the head model). For each participant, we generated a head model using a single-shell model constructed from the 
segmentation of the cortical tissue obtained from individual MRI scans (Nolte, 2003). Lead fields were not normalized. Sources were placed in the single-subject volumetric parcellation regions. For each region, the number of sources $n S P$ was computed as the ratio of the volume and the volume of a sphere of radius equal to $3 \mathrm{~mm}$. The $k$-means algorithm (Tou and Gonzalez, 1974) was then used to partition the 3D coordinates of the voxels within a given volumetric region into $n S$ clusters. The sources were placed at the center of each partition for each brain region. The head model, source locations and the information about MEG sensor position for both models were combined to derive single-participant lead fields. The orientation of cortical sources was set perpendicular to the cortical surface, whereas the orientation for subcortical sources was left unconstrained.

Adaptive linear spatial filtering (Van Veen et al., 1997) was used to estimate the power at the source level. The dynamical imaging of coherent sources (DICS) method was used, a beam-forming algorithm for the tomographic mapping in the frequency domain (Gross et al., 2001), which is well suited for the study of neural oscillatory responses based on single-trial source estimates of band-limited MEG signals (for a series of reviews, see Hansen et al., 2015). At each source location, DICS uses a spatial filter that passes activity from this location with unit gain while maximally suppressing any other activity. The spatial filters were computed on all trials for each time point and session and then applied to single-trial MEG data. DICS allows the estimate of complex-value spectral measures at the source level, $X_{\text {source }}^{n}(t, k)=A(t) X_{\text {sensor }}^{n}(t, k)$, where $A(t) \mathrm{s}$ the spatial filter that transforms the data from the sensor to source level and $X_{\text {sensor }}^{n}(t, k)$ is the complex-valued estimates of spectral measures, including cross-spectral density matrices, computed at the sensor level for each trial $n$, time $t$, and taper $k$ (for a detailed description of a similar approach, see Hipp et al., 2011). The single-trial high-gamma power at each source location was estimated by multiplying the complex spectral estimates with their complex conjugate, and averaged over tapers $k, P_{\text {source }}^{n}(t)=\left\langle X_{\text {source }}^{n}(t, k) X_{\text {source }}^{n}(t, k)_{k}^{\star}\right\rangle$, where angle brackets refer to the average across tapers and ${ }^{*}$ to the complex conjugate. Single-trial power estimates aligned on movement and stimulus onset were log transformed to make the data approximate Gaussian and low-pass filtered at $50 \mathrm{~Hz}$ to reduce noise. Single-trial mean power and SD in a time window from -0.5 and -0.1 s before stimulus onset was computed for each source and trial and used to $z$-transform single-trial movement-locked power time courses. Similarly, single-trial stimulus-locked power time courses were $\log$ transformed and $z$-scored with respect to baseline period to produce HGAs for the prestimulus period from -1.6 to $-0.1 \mathrm{~s}$ with respect to stimulation for subsequent FC analysis. Finally, single-trial HGA for each brain region of MarsAtlas was computed as the mean $z$-transformed power values averaged across all sources within the same region.

SEEG localization and HGA. Electrodes were localized using the CTMR toolbox (Hermes et al., 2010). Briefly, postimplant CT scans were coregistered and resliced to the MRI coordinate scans of each subject using SPM12. A manual procedure was then performed to mark the electrodes in the co-registered CT space using the CTMR toolbox. The coordinates of each electrode were transformed to MRI space ( $1 \mathrm{~mm}$ resolution). Because bipolar derivations were used, the coordinates of the midpoint between pairs of adjacent electrodes were computed. A cube of $5 \mathrm{~mm}$ in size was placed at these positions (i.e., at the position of the bipolar derivation) and each voxel of the cube ( $1 \mathrm{~mm}$ resolution) was labeled according to MarsAtlas. The location of each bipolar derivation was then labeled according to the label associated to the largest number of voxels within the cube. Bipolar derivations labeled in the white matter were excluded from further analyses.

Similarly to MEG HGA estimation, single-trial power estimates in the high-gamma range $(60-120 \mathrm{~Hz})$ aligned on movement and stimulus onset were log transformed and low-pass filtered at $50 \mathrm{~Hz}$ to reduce noise. Single-trial estimates of high-gamma power were $z$-transformed with respect to baseline period from -0.5 and $-0.1 \mathrm{~s}$ before stimulus onset. Finally, single-trial HGA for each labeled brain region of MarsAtlas was defined as the mean $z$-transformed power values averaged across all electrodes within the same region.

\section{Single-trial FCD measures}

Power modulations in the high-gamma range reflect the activity of local neural populations (Ray et al., 2008; Ray and Maunsell, 2011). Here, we assume that tracking statistical dependencies between HGA from different brain regions provides information about how local processing units coordinate at the large-scale level during cognitive tasks. The goal is not infer the mechanisms mediating interareal communication. This would require complementary approaches based on the study of role of neural oscillations and synchrony for interregional communication (Fries, 2015; Buzsáki and Schomburg, 2015). Rather, the aim was to map taskrelated FCD onto anatomical circuits. Given the sparseness of brain regions sampled with SEEG, FCD was performed exclusively for wholebrain MEG data.

Linear correlation analysis was used to study the FC between brain regions. To quantify the evolution of FC over time (i.e., FCD), the Pearson's correlation coefficient between pairs of HGA signals over sliding windows of $500 \mathrm{~ms}$ stepped every $10 \mathrm{~ms}$ was computed. The same procedure was performed across all pairs of brain regions and for each trial. This resulted in a 4 D FCD matrix (i.e., regions $\times$ regions $\times$ time points $\times$ trials) representing the evolution of linear correction across all pairs of brain areas from -0.7 to $0.7 \mathrm{~s}$ around movement onset. The single-trial FCD matrix was also computed during the prestimulus period, from -0.8 to $-0.1 \mathrm{~s}$ before stimulus onset for baseline. Statistical analyses searched for significant modulations in movement-related FCD with respect to those in the prestimulus interval.

\section{Statistical analysis}

Linear mixed effect (LME) model. Statistical inference of single-trial HGAs was performed using an LME model approach at the group level. A LME model was used because they are particularly suited for the analysis of data collected from multiple subjects (or sessions) for which it is important to take into account interindividual variability. These models formalize the relation between a response variable and independent variables using both fixed and random effects. Fixed effects model the response variable in terms of explanatory variables as nonrandom quantities. For example, experimental conditions related to population mean may be considered as fixed effects. Random effects are associated with individual experimental units drawn at random from a population, which may correspond to different participants in the study (or experimental sessions). In other words, whereas fixed effects are constant, random effects are drawn from a prior known distribution. A LME model is generally expressed in matrix formulation as follows:

$$
y=X \beta+Z b+e
$$

where $y$ is the $n$-by- 1 response vector and $n$ is the number of observations, $x$ is an $n$-by- $p$ fixed-effects design matrix, and $\beta$ is the fixed-effect vector of $p$-by- 1 , where $p$ is the number of fixed effects, $z$ is an $n$-by- $q$ random-effects design matrix, and $b$ is a $q$-by- 1 random-effects vector, where $q$ is the number of random effects and $e$ is the $n$-by- 1 observation error. The random-effects vector, $b$, and the error vector, $e$, were assumed to be drawn from independent normal distributions. Parameter estimation was performed using maximum likelihood method, using the fitlme.m function in the Statistical Toolbox of MATLAB (The MathWorks). To test for significant modulations in single-trial HGA and FCD measures around movement onset with respect to the baseline period, a random-intercept and random-slope LME model was used as described by the following:

$$
y(t)=\beta_{0}(t)+\beta_{1}(t) x_{j}+b_{0 j}(t)+b_{1 j}(t) z_{j}+\varepsilon_{j}(t)
$$

where $y(t)=\left[y_{b l}(1), y_{b l}(2), \ldots, y_{b l}(n p), y_{m v}(1, t), y_{m v}(2, t), \ldots, y_{m v}(n p, t)\right]$.

For MEG data analysis, $y_{b l}(j)$ was a vector containing the baseline neural activity (i.e., the HGA from single brain regions or FCD values for single pairs of regions) for all trials and sessions (i.e., data from both sessions were concatenated, because they were acquired in uninterrupted succession) for subject $j=1,2 \ldots n p$, where $n p$ is the number of participants at time instant $t$. Note that $t$ does not refer to trials, but rather the 
time within each trial. $y_{m v}(j, t)$ was a vector including brain activity across all trials for subject $j$ at time $t$ with respect to movement onset.

For SEEG data analysis, statistical inference was performed at the single-participant level due to the limited number of patients and limited sampling of MarsAtlas regions. However, given that the SEEG experiments were composed of two sessions acquired at different times $(\sim 1 \mathrm{~h}$ interval), we modeled sessions as random effects. $y_{b l}(j)$ was then a vector containing the baseline neural activity (i.e., the HGA from single brain regions) for all trials for sessions $j=1,2$ at time instant $t$. As before, $t$ did not refer to trials, but time within each trial. $y_{m v}(j, t)$ was a vector including brain activity across all trials for session $j$ at time $t$ with respect to movement onset.

The following statistical analysis was similar for both MEG and SEEG data. The design matrices contain two columns. The first column is a vector of ones to model the intercept, and thus it was eliminated from Equation 2. The second column contains negative ones for baseline trials and ones for event-related trials, therefore modeling the change with respect to baseline, or slope, and it is referred as $x_{\mathrm{j}}$ and $z_{\mathrm{j}}$ in Equation 2. Therefore, the first and third terms in the right side of Equation 2 model the intercepts, which correspond to the mean values between baseline and movement-related activity. The second and fourth terms model the slopes, which are the differences between baseline and movement-related activity. The $\beta_{1}(t)$ values are fixed across subjects, whereas the $b_{1 \mathrm{j}}(t)$ values model the random variations across subjects (for MEG) or sessions (for SEEG). In other words, the parameter $\beta_{1}(t)$ models the change in neural activity (e.g., HGA or FCD for MEG data) with respect to baseline at each time point $t$ at the group level; the parameter $b_{1 \mathrm{j}}(t)$ models the change in neural activity with respect to baseline for each participant (or sessions) $j$ and therefore explains the across-subjects (or across-session) variability for MEG and SEEG data, respectively. The across-subject and across-session variability was considered of no interest for the scope of the current analyses. We thus analyzed fixed effects. Given the structure of the fixed-effect design matrix, significant differences in movement-related neural activity with respect to baseline can thus be inferred by testing whether $\beta_{1}$ coefficients are significantly greater than zero. More formally, the significance of movement-related modulations was inferred using a $t$ test by testing the null hypothesis $H_{0}$ : $\beta_{1} \leq 0$.

Statistical inference was performed for each time point $t$ and each brain area for the analysis of HGAs. To account for the multiplecomparisons problem, the false discovery rate (FDR) was controlled (Benjamini and Yosef, 1995). For mean HGA statistical analyses, the number of time points and brain regions were corrected for; for FCD analyses, the number of time points and for the number of pairs of brain regions were corrected for. To further assess the validity of our results, the minimum number of consecutive significant time points required to reject a null hypothesis of absence of a cluster given a chance probability $p_{0}=0.5$ (two possible outcomes, significant or nonsignificant) were quantified and only those clusters with a duration that exceeded a given significance level were kept. Details of the calculation are given in the appendix of Smith et al. (2004).

The statistical analyses of MEG HGA modulations resulted in a grouplevel FCD matrix containing time-evolving $t$ - and $p$-values for each brain region in MarsAtlas (whole-brain analysis). For brain regions covered by the SEEG implants, the analysis of HGA modulations produced intracranial validation at the single-participant level. The analysis of FCD from MEG HGA produced $t$ - and $p$-value time courses for all pairs of brain regions.

\section{Graph theoretical analysis}

Strength of functional link (SFL). To gain insight into the topology of the task-related functional network arising from group-level MEG analyses, graph theoretical analyses of the FCD matrix containing the $p$-values associated with the LME analysis were performed. The weight or strength of the evidence of a functional link was defined as the minimum Bayes factor (BF) associated with such $p$-values (Goodman, 1999b). The rationale behind the transformation of $p$-values to minimum BFs is an attempt to move toward statistical measures that can be better interpreted (Goodman, 1999a). The BF is a convenient measure of the strength of statistical evidence and it can be computed from $p$-values as $B F_{u p}<-1 /$ $(e p \ln (p))$ if $p$-values satisfy the relation $p<1 / e$, in which $e \approx 2.72$. This estimate provides an upper bound $\left(B F_{\text {up }}\right)$ on the $\mathrm{BF}$, and it can be thought as providing an "optimistic" limit of the BF for a given $p$-value (Goodman, 2001; Stephens and Balding, 2009). BFs were log transformed to give a measure that quantifies the strength in the evidence of the presence of a functional link between two brain regions, $S F L=\log _{10}$ $B F_{u p}$. The $S F L$ matrix has the same dimensions of group-level FCD matrix (i.e., regions $\times$ regions $\times$ time points). A value between 1 and 2 can be interpreted as providing strong to very strong evidence of a functional link (i.e., increase in correlation with respect to baseline), whereas a value $>2$ is interpreted as decisive.

Analysis of time-averaged and time-dependent SFL. A caveat of FC analysis is the dependence of functional links on the threshold chosen for statistical significance. Therefore, to explore how graph theoretical measures vary according to significance levels, the SFL matrix was multiplied with different binary masks obtained from the FDR correction of the FCD matrix over a wide range from highly significant values $\left(p_{\text {FDR-corrected }}=q<0.001\right)$ to nonsignificant $(q<0.99999)$. Note that a FDR-adjusted $p$-value is denoted as a $q$-value. This produced several thresholded SFL matrices, each one associated with a given level of significance.

As a first analysis, the mean SFL matrices were computed over time, thus giving an adjacency matrix (regions $\times$ regions) representing the mean strength between brain regions at different significance levels. The density $D$ (the ratio between the number of functional links and the number of possible connections) was computed as a function of the $q$-value. In addition, to identify the most important brain regions in average SFL graph, the strength of each region (sum of functional links of a region) and two indicators of centrality, such as the eigenvector centrality $E C$, defined as the absolute value of the eigenvector associated with the largest eigenvalue of the adjacency matrix $\mathrm{W}$ and it measures the importance of a region, and the between-ness centrality $(B C)$, which is equal to the fraction of all shortest paths that pass through a given region, so it measures the number of times a region that acts as a "bridge" were computed. These measures were, however, computed only at $q<0.05$. Finally, to evaluate the evolution of density of the thresholded FCD, it was computed for each time slice rather over the averaged FCD. Graph theoretical measures were computed using the Brain Connectivity Toolbox (Rubinov and Sporns, 2010).

Detection of functional subnetworks. A critical step in the analysis of brain networks is the detection of communities, which may correspond to functional subnetworks. Subnetworks, however, may overlap spatially, such that a given brain region may belong to more than one group. Link communities, defined as groups of links rather than nodes, provide an appropriate framework for capturing the relationships between overlapping communities while revealing hierarchical organization (Ahn et al., 2010). To detect time-varying link communities, an approach based on the analysis of the correlation of edge weights over time, rather than nodes, was used, similarly to previous works analyzing "cross-links" or "hyper-edges" (Bassett et al., 2014; Davison et al., 2015).

First, the Pearson linear correlation between significant (at $q<0.05$ ) pairs of SFL time courses was computed. This produced an adjacency matrix (number of links in size) representing the temporal correlation between functional links. Second, the optimal subdivision of such graph into groups of links was searched for using the Louvain method (Blondel et al., 2008), which attempts to optimize the "modularity" of a partition of the network. The Louvain algorithm for modularity maximization is a nondeterministic heuristic, so it needs to be initialized with random seeds. In addition, it depends on the resolution parameter $\gamma$, which controls over the size and number of communities found (resolution equal to 1 leads to the standard Louvain method, whereas higher and lower resolutions produce larger and smaller number of clusters, respectively). We scanned different resolution parameters from $\gamma=0.5$ to $\gamma=1.5$ in increments of 0.1. At each scale, we ran the Louvain method 250 times to test whether the nondeterministic nature of the method could produce nonrobust results. For all pairs of partitions $\left(250^{\star} 249\right.$ in total), similarity, defined as the $z$-score of the Rand index (Traud et al., 2011) was computed and averaged across all pairs of partitions. The optimal resolution 


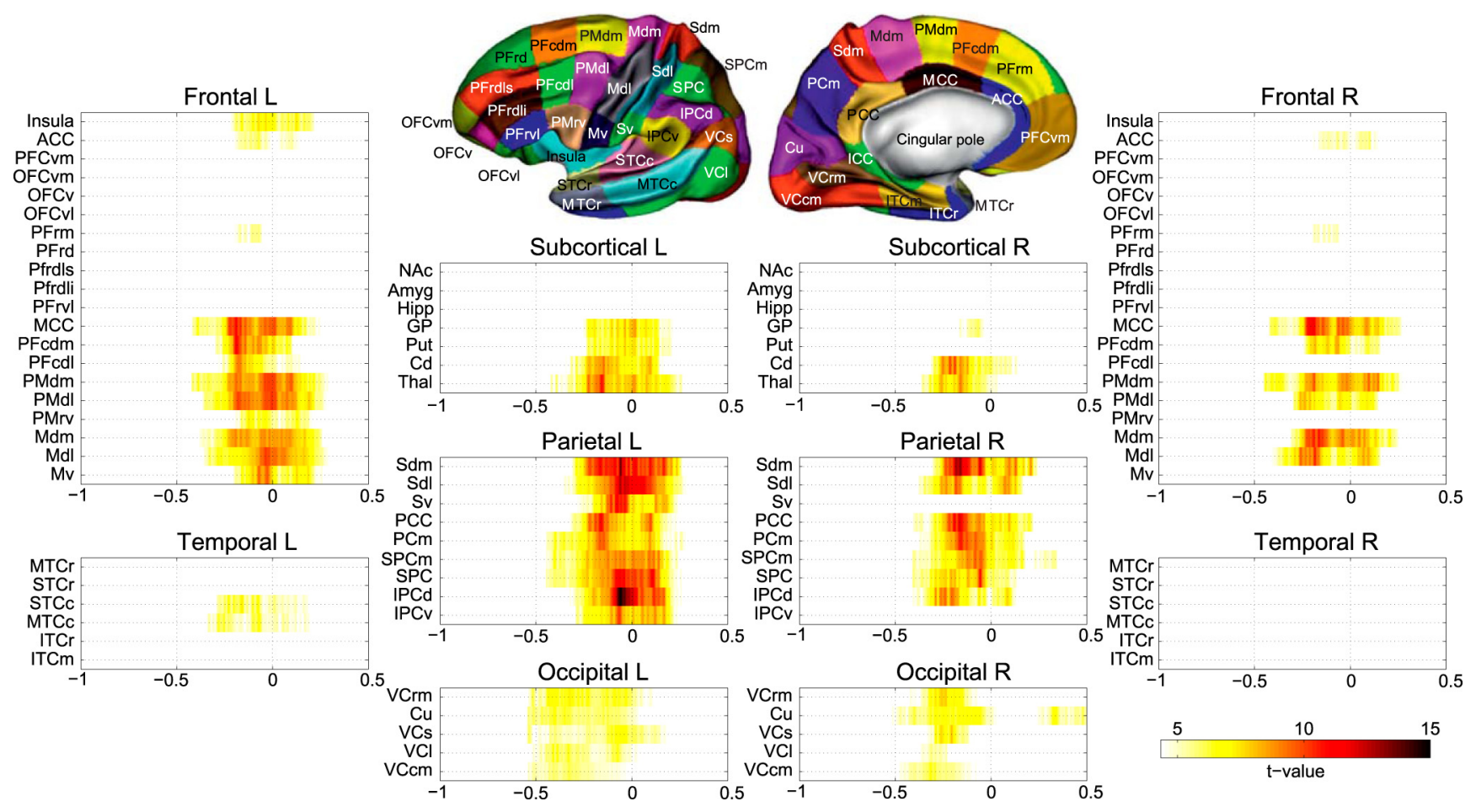

Figure 2. Statistical map displaying the brain areas associated with a significant increases in HGA with respect to baseline (time point and cluster-level threshold were set to $q<0.001 \mathrm{FDR}$ corrected). The anatomical labels of subcortical areas are NAc (nucleus accumbens), Amyg (amygdala), Hipp (hippocampus), GP (globus pallidus), Put (putamen), Cd (caudate nucleus), and Thal (thalamus).

parameter $\gamma$ was associated with the largest average similarity between partitions. The largest similarity was observed at $\gamma=1$. For $\gamma=1$, the consensus partition was studied to identify a single representative partition from a set of 250 partitions based on statistical testing compared with a null model. The representative partition is obtained by using a generalized Louvain algorithm on the thresholded nodal association matrix (Bassett et al., 2013). For our FCD matrix at $\gamma=1$, the Louvain algorithm is extremely stable and the 250 partitions are all identical. These graph theoretical analyses were performed using the "Consensus and Comparison Methods" in the Network Community Toolbox (http://commdetect.weebly.com/).

This approach provides a subdivision of nonoverlapping communities by maximizing the number of within-group edges and minimizing the number of between-group edges. Given that community detection was performed on links, the detected communities represent link communities in which individual brain areas may participate in multiple overlapping networks. Finally, the mean time course of the SFL averaged across all links comprising each link community was computed.

\section{Results}

\section{Visuomotor-related functional network}

The brain regions displaying a significant increase in movementrelated HGA with respect to the mean baseline (averaged from -0.5 to $-0.1 \mathrm{~s}$ before stimulus onset) defined the arbitrary visuomotor-related network (Fig. 2) For cortical regions, the largest increase in HGA was observed over the left parietal lobe, primarily over the dorsal (dorsal intraparietal cortex, IPCd, and the superior parietal cortex, SPC) and medial (medial superior and medial parietal cortices, SPCm and PCm, respectively) parietal regions, the dorsal somatosensory areas (Sdl and Sdm) and the posterior cingulate cortex (PCC). The ventral regions, such as (IPCv and Sv), displayed a smaller increase relative to the dorsal and medial territories in the left hemisphere and were not significant in the right hemisphere. Over the motor, premotor, and prefrontal areas, the dorsolateral and dorsomedial regions
(PFcdl, PFcdm, PMdl, PMdm, Mdl and Mdm) showed the most significant increase. In addition, the midcingulate cortex (MCC) showed significant response bilaterally. The ventral and ventromedial prefrontal and orbitofrontal cortices did not display a strong increase in HGA nor anterior temporal regions. These cortical modulations are similar to those presented in a previous study (Auzias et al., 2016), but we replicate them here for completeness.

The novel finding, however, is the presence of significant HGA modulations in subcortical areas. The strongest response was observed in the left hemisphere in the dorsal striatum (caudate nucleus and putamen), globus pallidus (GP) and thalamus (Thal). The thalamus and caudate nucleus displayed a clear bilateral activation, whereas the GP and putamen showed primarily an activity in the hemisphere contralateral to the motor response. A significant response was also observed in the right thalamus and caudate nucleus. No significant increase was seen in other subcortical areas examined such as the nucleus accumbens, amygdala, and hippocampus.

\section{Visuomotor-related FCD}

The analysis of FCD between all pairs of brain regions of MarsAtlas was performed by estimating Pearson's correlation coefficients between pairs of single-trial HGA values over sliding windows of $500 \mathrm{~ms}$ stepped every $10 \mathrm{~ms}$. For each participant, FCD analysis resulted in a 4D matrix (i.e., regions $\times$ regions $\times$ time points $\times$ trials) representing the evolution of linear correlation across all pairs of brain areas from -0.7 to $0.7 \mathrm{~s}$ around movement onset. Significant modulations in movement-related FCD with respect to those in the baseline period from -0.8 to $-0.1 \mathrm{~s}$ before stimulus onset using an LME approach. Figure $3 \mathrm{~A}$ shows the connectivity matrix of the average SFL over time. Note that $p$-values were thresholded at $q<0.05$ (FDR-corrected) be- 
A

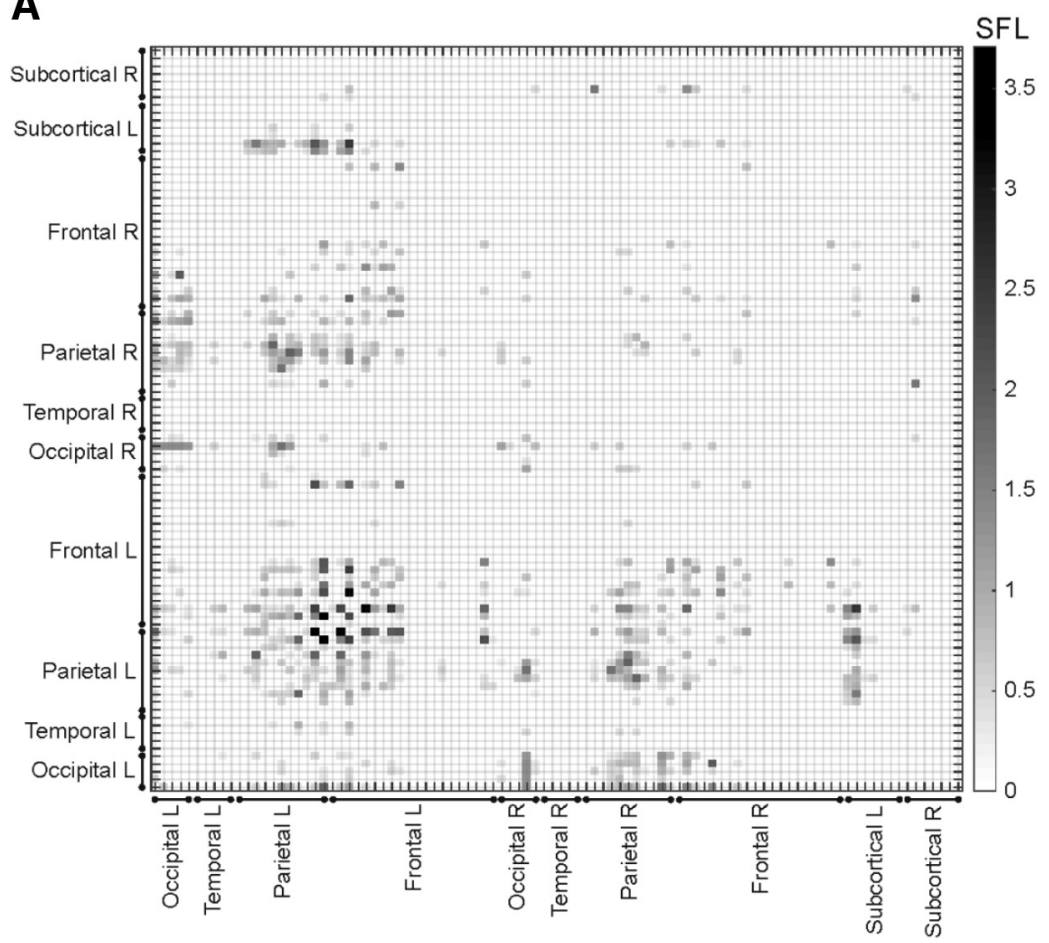

B

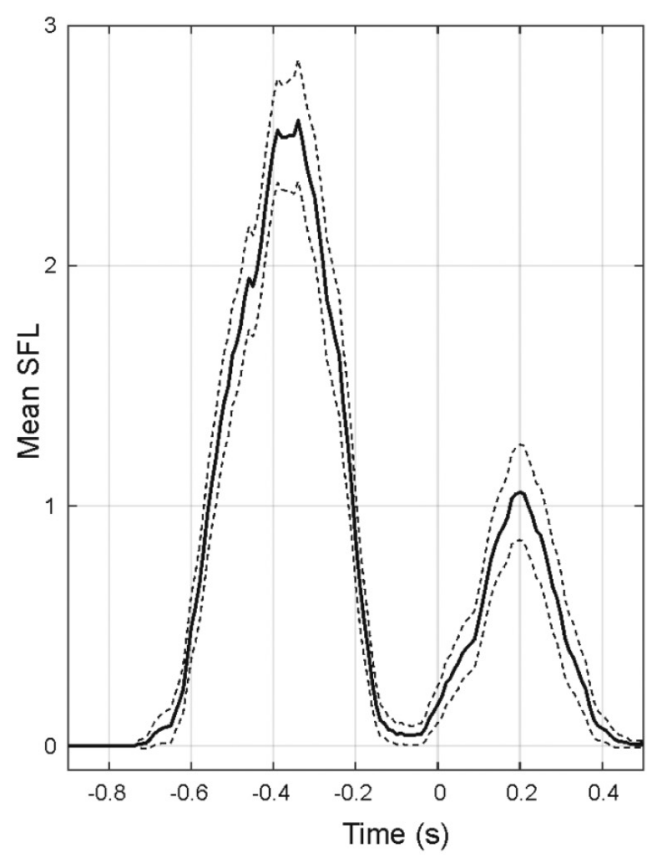

Figure 3. SFL.A, Mean SFL connectivity matrix averaged over time. B, SFL time course averaged over pairs of areas. The threshold for significant SFL was equal to 2.43 . Error bars indicate the $95 \%$ confidence interval.

fore SFL computation, which corresponds to a threshold value of SFL equal to 2.43. All significant links shows decisive evidence in FC averaged over time among occipital areas bilaterally with strong links with parietal regions, in addition to the frontoparietal network. Subcortical regions, especially in the left hemisphere, showed a strong FC with the rest of the network. Regions in the temporal lobes, however, were not found to play a key role in the FC patterns.

To better characterize the evolution of the SFL over time, we computed the mean SFL across pairs of brain regions displaying a significant increase in linear correlation (Fig. 3B). The mean time course displays two peaks of decisive and strong evidence at $\sim-0.4$ and $0.2 \mathrm{~s}$ around finger movement. The time intervals around the two peaks represent moments when FC pattern is strongest, which correspond to the largest increase in linear correlation between HGA time courses. Given the shape of the group-level HGA responses shown in Figure 2, the first peak at $-0.4 \mathrm{~s}$ reflects the positive covariation in HGA across the whole network occurring after stimulus presentation and during movement planning (as early as -0.55 in visual areas to $\sim-0.25 \mathrm{~s}$ before finger movement). Such a common increase in HGA produces an increase in linear correlation and reflects the emergence of the FC network. The second peak occurring at $0.2 \mathrm{~s}$ reflects a common return to baseline of the HGA across the whole network after finger movement (from $\sim 0.05 \mathrm{~s}$ to $0.35 \mathrm{~s}$ after finger movement). Such a global decrease in HGA from maximal activity produces an increase in linear correlation, but reflects the dissolution of the FC network. Therefore, the two peaks correspond to the emergence and dissolution of the FC pattern. The decrease in FC occurring $\sim-0.12 \mathrm{~s}$ to $-0.03 \mathrm{~s}$ before finger movement corresponds to the positive peak of HGA (Fig. 2). Such a decrease in FC, therefore, does not reflect an absence of HGA, but rather a maximum of HGA. However, it corresponds to the time interval when FC lacks any significant covariation. Overall, the analysis of the FCD time course reveals two key processes such as the creation and dissolution of FC network.

\section{Graph theoretical analysis of FC network}

To gain insight into the properties of the average FC pattern, we performed graph theoretical analyses of the average SFL matrix shown in Figure $4 A$. We investigated graph theoretical measures of the mean SFL matrices averaged over time at different thresholds $(q<0.001)$ to nonsignificant $(q<0.99999)$. Figure $4 A$ shows the FC network density $D$ (the ratio between the number of functional links and the number of possible connections) as a function of threshold $q$-values. The density of the functional network trivially increase as a function of the threshold; that is, the more functional links, the higher is the density. The density values for significant $q<0.05$ are $<10 \%$, meaning that the functional network is not dense, but sparse. We then computed the density for each time slice of the FCD. Network density is maximal $(\sim 5 \%) \sim 0.4 \mathrm{~s}$ and then displays a second peak 0.2 after finger movement ( $2 \%$ in density), as shown in Figure $4 B$.

Finally, to identify the most important brain regions in the average SFL graph, we computed the strength $S$ (sum of functional links of a region), the eigenvector centrality $E C$, and the $B C$ at $q<0.05$. The strength of a brain area is the simplest measure to estimate the importance of a node in a network. A natural extension of strength centrality is eigenvector centrality EC and it stands on the notion that a node is important if it is linked to by other important nodes. In fact, a node receiving many links (i.e., high strength) does not necessarily have a high eigenvector centrality because it may be linked to node with low strength. Therefore, EC provides additional information because it computes the centrality of a node as a function of the centralities of its neighbors. Finally, the $B C$ is equal to the number of shortest paths that pass through a brain region. Therefore, a region with high $B C$ has the potential to play a key role in the network. Convergence of 
A

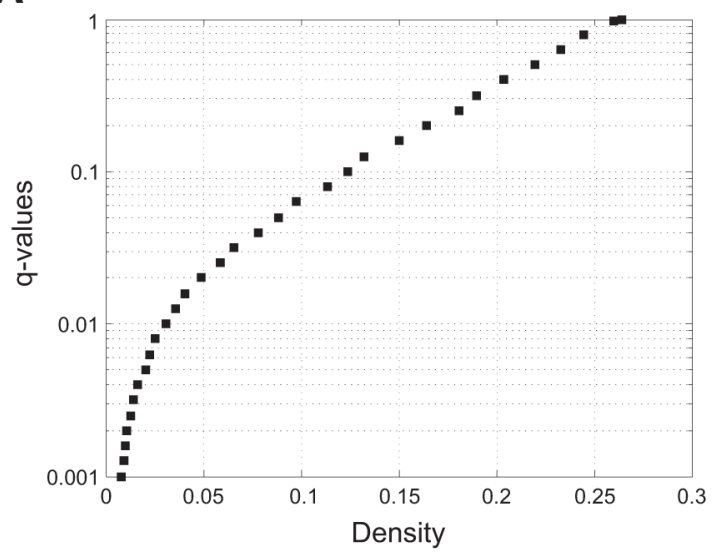

B

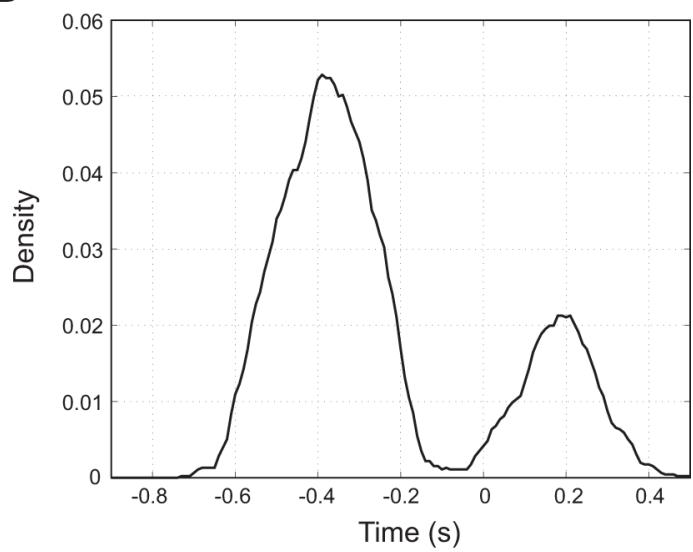

Figure 4. Graph theoretical measures. $\boldsymbol{A}$, Dependence between threshold values ( $q$-values) and FC density. $\boldsymbol{B}$, Temporal evolution of density $D$ at $q=0.05$.

Table 1. Graph theoretical measures

\begin{tabular}{|c|c|c|c|c|c|}
\hline \multicolumn{2}{|l|}{$S$} & \multicolumn{2}{|l|}{$E C$} & \multicolumn{2}{|l|}{$B C$} \\
\hline Value & Region & Value & Region & Value & Region \\
\hline 7.39 & Mdm & 6.84 & Mdm & 16.25 & Sdm \\
\hline 6.28 & Sdm & 6.42 & Sdm & 13.68 & Mdm \\
\hline 4.64 & Sdl & 5.73 & Sdl & 9.98 & $\mathrm{Cu}$ \\
\hline 3.88 & PMdl & 4.98 & Mdl & 9.52 & $\mathrm{VCcm}$ \\
\hline 3.75 & $\mathrm{VCcm}$ & 4.37 & PMdl & 6.19 & Sdl \\
\hline 3.70 & Mdl & 3.82 & $\mathrm{Cd}$ & 5.81 & Mdl \\
\hline 3.34 & SPC & 3.56 & VCcm & 4.80 & PMdl \\
\hline 3.25 & $\mathrm{SPCm}$ & 3.37 & $\mathrm{SPCm}$ & 4.46 & $I P C d$ \\
\hline 3.20 & $\mathrm{Cu}$ & 3.20 & PFcdm & 3.83 & SPC \\
\hline 3.13 & $\mathrm{Cd}$ & 3.05 & $P C C$ & 3.41 & $\mathrm{SPCm}$ \\
\hline
\end{tabular}

these three metrics provides information about the importance of different brain regions in the network.

Table 1 shows the brain regions sorted in a descending order according to $S, E C$, and $B C$. The brain areas that commonly emerge as relevant across the three measures are the dorsomedial and dorsolateral sensorimotor regions ( $\mathrm{Mdm}$, $\mathrm{Mdl}, \mathrm{Sdm}$ ), in addition to dorsolateral premotor area (PMdl), superior parietal regions (SPC, SPCm), and the caudomedial visual cortex $(\mathrm{VCcm})$.

\section{Dynamic reconfiguration of FC subnetworks}

To search for functional subnetworks generating the observe dynamics, we performed link community analysis. To do so, we first computed the Pearson linear correlation between significant (at $q<0.05)$ pairs of SFL time courses. Then, we found the optimal subdivision of such link graph into communities of links using an algorithm that attempts to optimize the "modularity" of a partition of the network, named the Louvain method (Blondel et al., 2008). This approach provides a subdivision of nonoverlapping communities by maximizing the number of within-group edges and minimizing the number of between-group edges. Given that community detection was performed on links, the detected subnetworks represent link communities, in which individual brain areas may participate in multiple overlapping networks. The analysis revealed the presence of three link communities (Fig. 5). The first link community (LC1) primarily included the visual and superior and medial parietal regions bilaterally, in addition to the left dorsomedial and dorsolateral sensorimotor regions (Fig. 5B). These brain regions form a FC subnetwork emerging $\sim 0.5 \mathrm{~s}$ before finger movement, approximately corresponding to the pro- cessing of the visual cue (Fig. 5A). The second link community (LC2) included the left dorsolateral and dorsomedial sensorimotor regions and the dorsal frontoparietal network. Interestingly, it included the middle and anterior cingulate cortices, the dorsomedial prefrontal cortex, and the dorstal striatum in the caudate nucleus (Fig. 5C). LC2 emerged later during the trial and its maximum of expansion occurred $\sim 100-150 \mathrm{~ms}$ after LC1, -0.35 s before movement. LC3 involved a larger brain network involving the bilateral sensorimotor regions, the left frontoparietal network, and visual areas (Fig. 5D). LC3 showed a strongest peak after finger movement at $0.2 \mathrm{~s}$, but displayed a peak at -0.4 s. The only regions of the temporal lobe showing significant FC were the superior and midtemporal cortices in the left hemisphere. However, these regions displayed a relative weak strength in the observed networks (Fig. 5B-D). Overall, the link community analyses allowed us to identify multiple and spatially overlapping FC patterns that evolve dynamically during the trial.

We can therefore depict the involvement of key brain regions in a given link community such as the motor and premotor areas. Figure 6 shows the contribution of the dorsolateral motor and dorsal premotor areas, Mdl and PMdl, respectively, in the three subnetworks as it unfolds over time. Around the presentation of the visual stimulus, the Mdl primarily participates in the $\mathrm{LC1}$, which gradually declines over time (blue curve in Fig. 6A,B). Then, its involvement in LC2 and LC3 increases in parallel, but peaks earlier for LC2 (red curve in Fig. 6A, B) rather than for LC3 (green curve). These curves depict the dynamic reconfiguration of the primary motor area from stimulus onset to motor output. For the dorsolateral premotor cortex, its contribution peaking for LC1, followed by LC3 and LC2 (Fig. 6C,D), confirms that the dynamic engagement in different subnetworks occurs over a short timescale.

Finally, to quantify the dynamics of reconfiguration among the three functional subnetworks, we inferred the evolution of network "flexibility" (following the same ideas developed in Braun et al., 2015). We defined the flexibility of a given brain region as the entropy associated with the probabilities of involvement in the three LCs (shown in Fig. $6 B, D$, for Mdl and PMdl, respectively). Accordingly, node flexibility is maximal for nodes participating with equal probability in the different LCs and minimal for nodes participating in a single LC. Figure $7 A$ shows the mean dynamics of network flexibility averaged over nodes. Interestingly, it reconfiguration shows a single peak occurring at $\sim 0.4$ $s$ before finger movement, which corresponds to the moment 
A

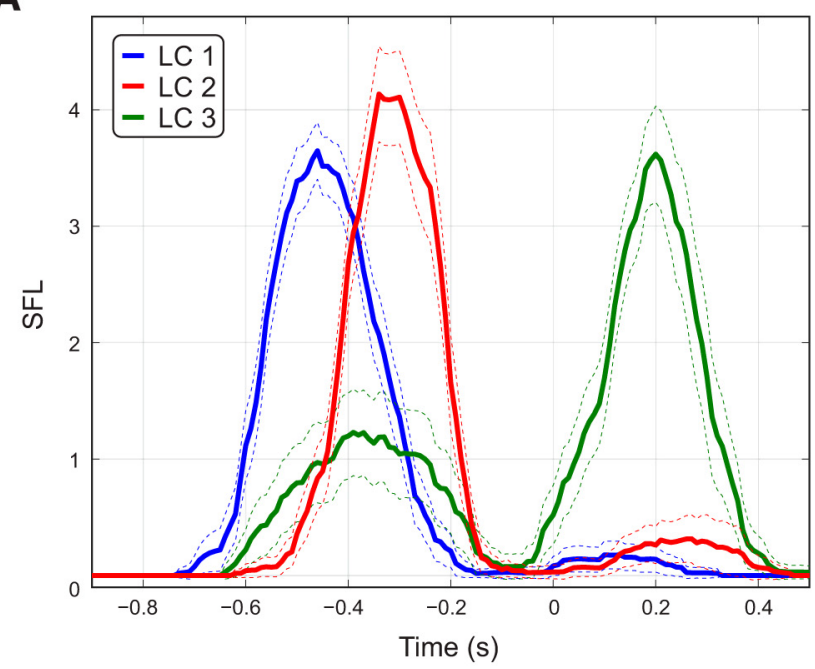

B

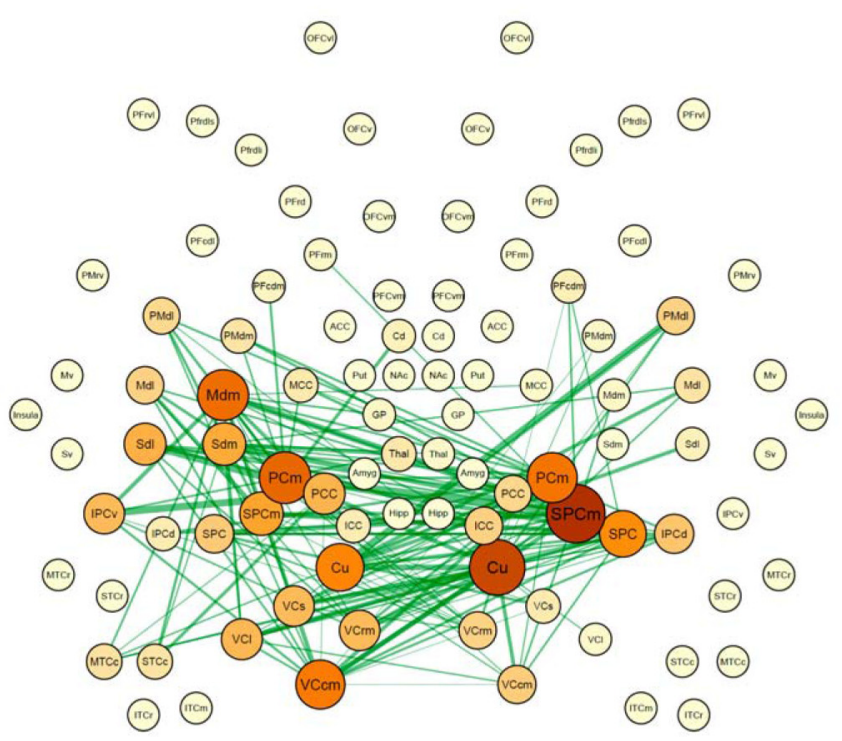

C

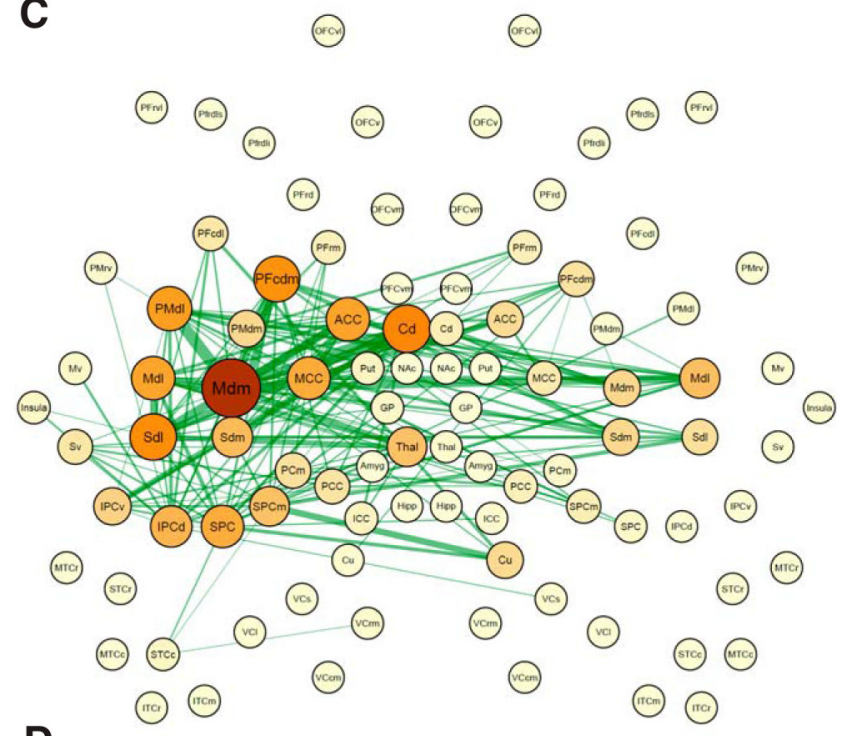

D

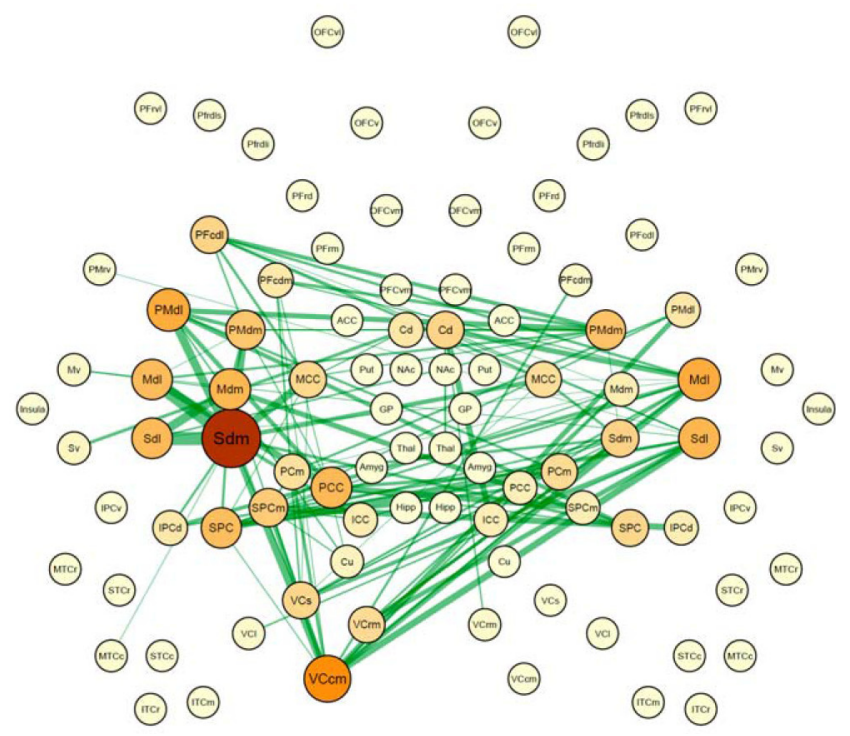

Figure 5. FCD. $\boldsymbol{A}$, Time course of the average SFL for the three identified LCs. Error bars indicate the $95 \%$ confidence interval. Spatial patterns for the three $L C$ s are displayed in $\boldsymbol{B}-\boldsymbol{D}$. The thickness of the green links is proportional to the time-averaged SFL between areas and the color and size of nodes are proportional to the mean SFL between each area and the rest of the network (i.e., the weight).

when the three LCs overlap more strongly in time and space. Figure $7 B$ shows the mean flexibility averaged over time for the first five strongest brain regions. The parietal regions (SPC, PCm, and PPC) display the largest flexibility, together with the dorsal premotor cortex.

\section{Control analyses and intracranial SEEG validation}

The interpretation of FC measures from noninvasive techniques such as EEG and MEG may suffer limitations, among which volume conduction and leakage are potential confounds (Bastos and Schoffelen, 2015). We performed a series of control analyses to assess the influence of such confounds.

First, we studied the relation between the mean SLF for two subcortical regions displaying a significant increase in HGA and FC with cortical regions as a function of their respective distance to cortical regions. The rationale was to investigate whether volume conduction and leakage effects could have produced the observed HGA modulations in subcortical regions and the FC patterns between deep sources, such as the thalamus and caudate nucleus and cortical areas. Figure 8 shows that a clear relation between distance and FC measures (as assessed through the mean SFL) is lacking for the thalamus (Fig. $8 A$ ) and for the caudate nucleus (Fig. 8B).

Second, we investigated the dipole orientation of all sources within the thalamus and caudate nucleus. The rationale was that differences in dipole orientation of nearby regions may suggest that the estimated HGAs originate from spatially separable brain structures. We computed the average dipole orientation both for the thalamus and caudate nucleus. Then, we compared the average dipole orientations by means of the normalized inner product. This measure equals one for identically oriented dipoles minus one for dipoles pointing in opposite directions and zero for orthogonal dipoles. The boxplot displayed in Figure 9A depicts the distribution of normalized inner products between the thalamus and caudate nucleus across participants. The values of normalized inner products span a broad range from -0.45 to 
A

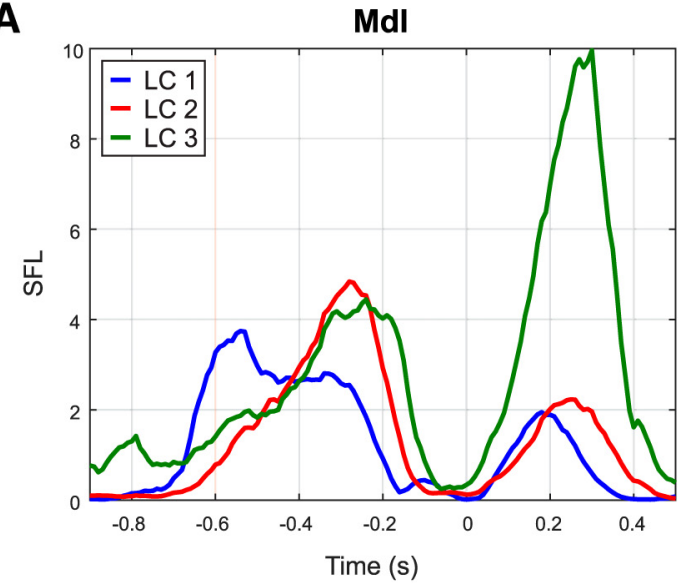

B

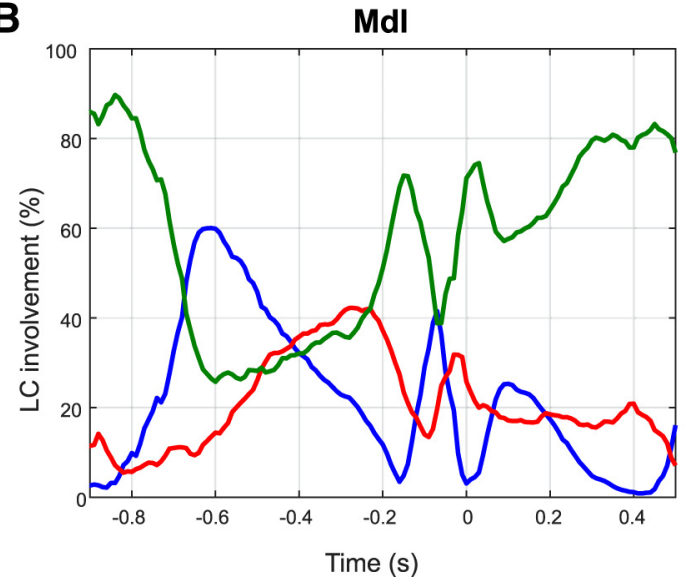

C

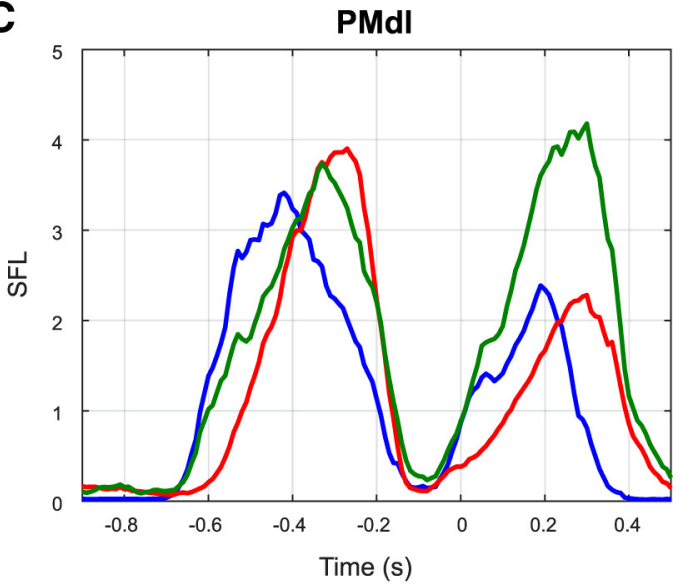

D

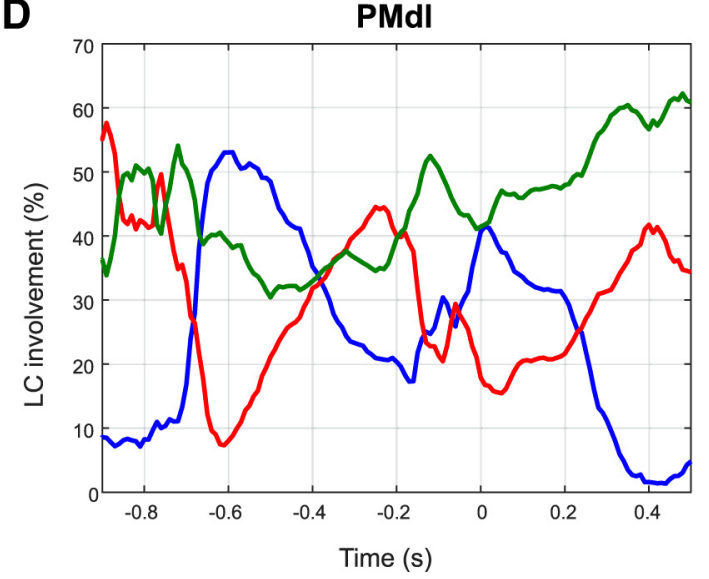

Figure 6. Exemplar evolution for the dorsolateral motor $(\boldsymbol{A}, \boldsymbol{C})$ and premotor $(\boldsymbol{B}, \boldsymbol{D})$ areas. $\boldsymbol{A}$ and $\boldsymbol{B}$ show the involvement of Mdl and PMdl in the three $L(C$, respectively. $\boldsymbol{C}$ and $\boldsymbol{D}$ depict the involvement in percentage value.

A

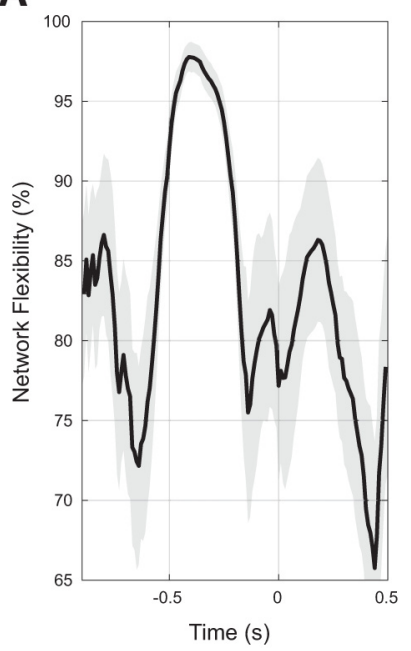

B

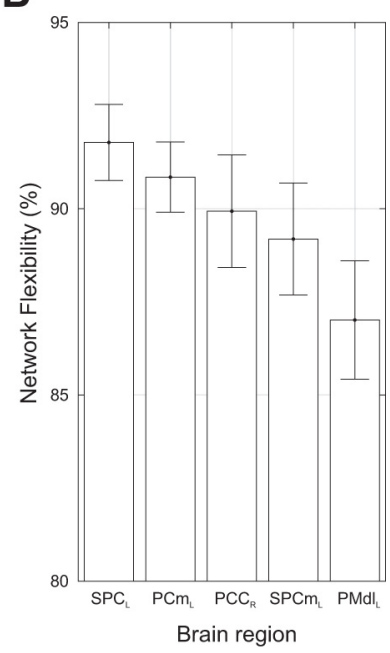

Figure 7. Flexibility analysis. $\boldsymbol{A}$, Mean evolution of network flexibility (shaded area represents the SD). $\boldsymbol{B}$, Areal flexibility for the five most representative brain regions.

0.85, with median value around 0.65 . Extremely high values of raw correlation coefficient between subcortical HGAs would also suggest strong leakage effects. We thus plotted the Pearson correlation coefficient between the HGA at the thalamus and caudate nucleus, averaged over sessions and participants (Fig. 9B).

The value in the prestimulus interval (i.e., $\sim-0.8 \mathrm{~s}$ before finger movement) was 0.615 . The corresponding coefficient of determination $R^{2}$ was $37,8 \%$ (i.e., $R^{2}=0.615 \times 0.615=0.3782$ ) and equals the proportion of the variance in HGA shared by the thalamus and caudate nucleus.

Finally, to assess the relevance of cortical HGA modulations, we asked three patients candidate for surgical treatment of drugresistant epilepsy to perform the same visuomotor task while recording SEEG in multiple brain areas. HGA from intracranial recordings in epileptic patients is largely exploited for cognitive mapping and represents an optimal opportunity to validate MEG results. Results from single-patient SEEG cannot be taken as representative of the population, contrary to group-level MEG results. In fact, we cannot exclude that across-subject variability is due to either physiological and/or pathological factors. However, SEEG data provide direct measures of intracranial HGA, free from alteration due to volume conduction or limitation of source imaging tools. Therefore, they provide important additional evidence to support the significance of the MEG results.

Electrodes were localized by combing postimplant CT scans with presurgical anatomical MRI scans and were labeled according to MarsAtlas (see Materials and Methods). Single-trial HGA for each labeled brain region of MarsAtlas was defined as the mean $z$-transformed power values averaged across all electrodes within the same region. To increase the local specificity of SEEG recordings, bipolar derivations were performed among adjacent contacts. Statistical analyses were performed separately for each 
A

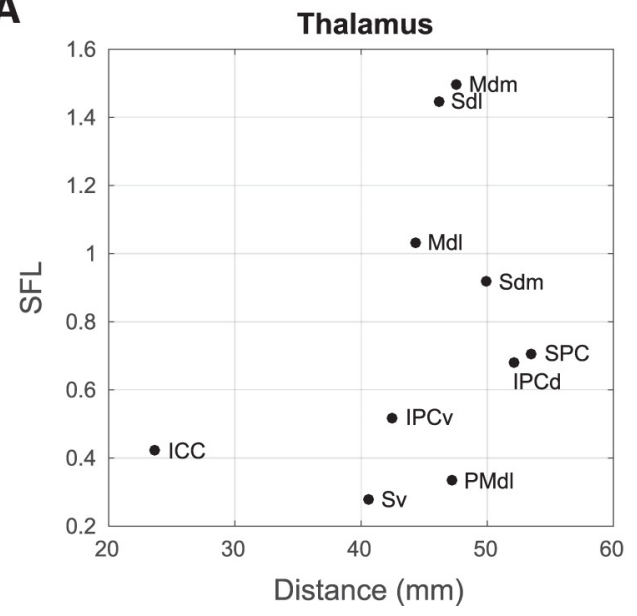

B

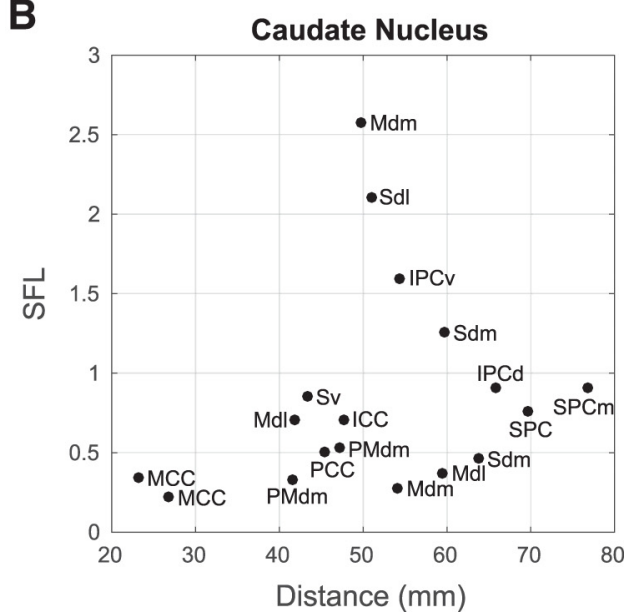

Figure 8. Relation between mean $\mathrm{FC}$ and distance for the thalamus $(\boldsymbol{A})$ and caudate nucleus $(\boldsymbol{B})$. Each dot corresponds to a brain area displaying significant $\mathrm{FC}$ with the seed regions.

A

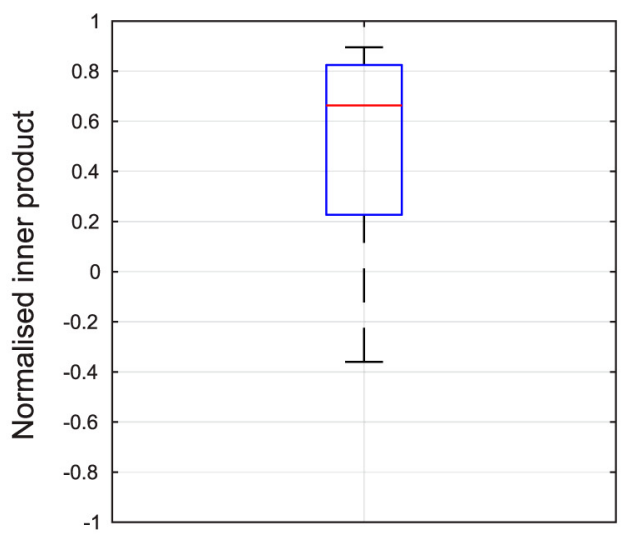

B

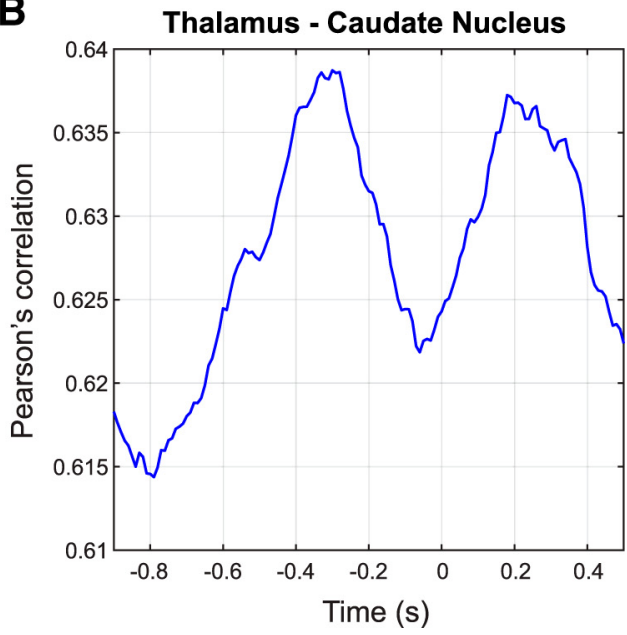

Figure 9. Distribution of normalized inner products between the average dipole orientations of the left thalamus and caudate nucleus across participants $(\boldsymbol{A})$. The boxplot depicts extreme values (whiskers), first and third quartile (box) and median (red line). B, Time course of mean correlation coefficient.

Table 2. SEEG activations

\begin{tabular}{lllllll}
\hline $\begin{array}{l}\text { Parcel } \\
\text { name }\end{array}$ & $\begin{array}{l}\text { Peak } \\
p \text {-value }\end{array}$ & $\begin{array}{l}\text { Peak } \\
t \text {-value }\end{array}$ & $\begin{array}{l}\text { Peak } \\
\text { time }(s)\end{array}$ & \multicolumn{2}{l}{ Time interval (s) } & $\begin{array}{l}\text { Patient } \\
\text { no. }\end{array}$ \\
\hline L Mdm & $3.42 \mathrm{E}-20$ & 11.43 & -0.175 & -0.28 & 0.125 & 1 \\
R SPC & $8.76 \mathrm{E}-20$ & 11.15 & -0.22 & -0.44 & 0.2 & 3 \\
R PMdm & $1.41 \mathrm{E}-17$ & 10.27 & -0.34 & -0.62 & -0.06 & 2 \\
L PMdm & $5.65 \mathrm{E}-17$ & 10 & -0.29 & -0.455 & 0.155 & 1 \\
R SPCm & $4.85 \mathrm{E}-17$ & 9.96 & -0.045 & -0.275 & 0.05 & 3 \\
L Mdl & $2.21 \mathrm{E}-15$ & 9.29 & -0.435 & -0.555 & -0.315 & 1 \\
R Mdl & $1.52 \mathrm{E}-14$ & 8.92 & -0.165 & -0.39 & 0.055 & 2 \\
R PMrv & $6.70 \mathrm{E}-14$ & 8.63 & -0.145 & -0.5 & 0.155 & 2 \\
LSdl & $1.29 \mathrm{E}-12$ & 8.05 & 0.045 & -0.225 & 0.26 & 1 \\
R IPCd & $1.69 \mathrm{E}-12$ & 7.96 & -0.28 & -0.415 & -0.135 & 3 \\
L PMdl & $4.22 \mathrm{E}-12$ & 7.82 & -0.315 & -0.45 & -0.185 & 1 \\
R Mv & $2.78 \mathrm{E}-11$ & 7.44 & -0.02 & -0.44 & 0.18 & 2 \\
R Mdl & $9.89 \mathrm{E}-08$ & 5.72 & -0.475 & -0.51 & -0.4 & 2 \\
\hline
\end{tabular}

patient. Twelve brain areas across the three patients were found to display a significant increase in HGA (Table $2 ; q<0.05$ ). These included the dorsomedial and dorsolateral motor cortex, the dorsolateral somatosensory region, and the dorsal frontoparietal network (SPC, SPCm, PMdl, and PMdm). In patient 2, the ventral portions of the motor and premotor areas were significantly active. Note that the SEEG implant did not cover the entire brain, but selected regions in the frontoparietal network.

We then compared the average time course of single-patient HGA modulations with those from the MEG group-level analyses (Fig. 10). Seven of 12 regions displayed a strong (larger than 0.65 ) linear correlation between the group-level MEG results and the single-patient SEEG time courses. The most striking similarity was observed for brain regions of the sensorimotor cortices and the dorsal frontoparietal network. Overall, these results confirm that the increase in HGA observed in the MEG data over sensorimotor cortices and the dorsal frontoparietal network results from area-specific increases in HGA, rather than by leakage from nearby regions displaying a strong response.

\section{Discussion}

Brain network and interactions of visuomotor mapping

Previous analyses of arbitrary visuomotor-related FC have shown that parietal areas play a driving role in the network, whereas premotor areas act as relays from parietal to medial prefrontal cortices, which participate as receivers (Brovelli et al., 2015). Such an approach, however, neither considered the time-evolving nature of FC patterns nor analyzed the involvement of subcortical areas. Our whole-brain and time-dependent brain connectivity 

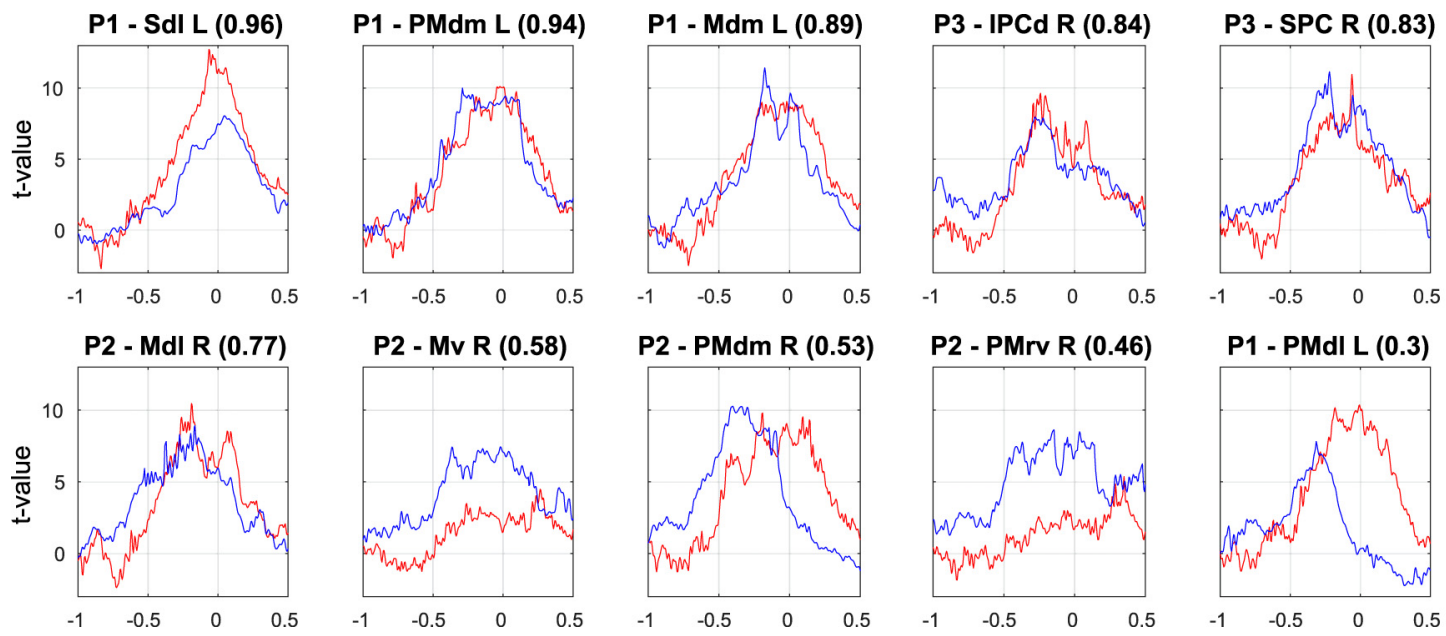

P3 - SPCm R (0.82)
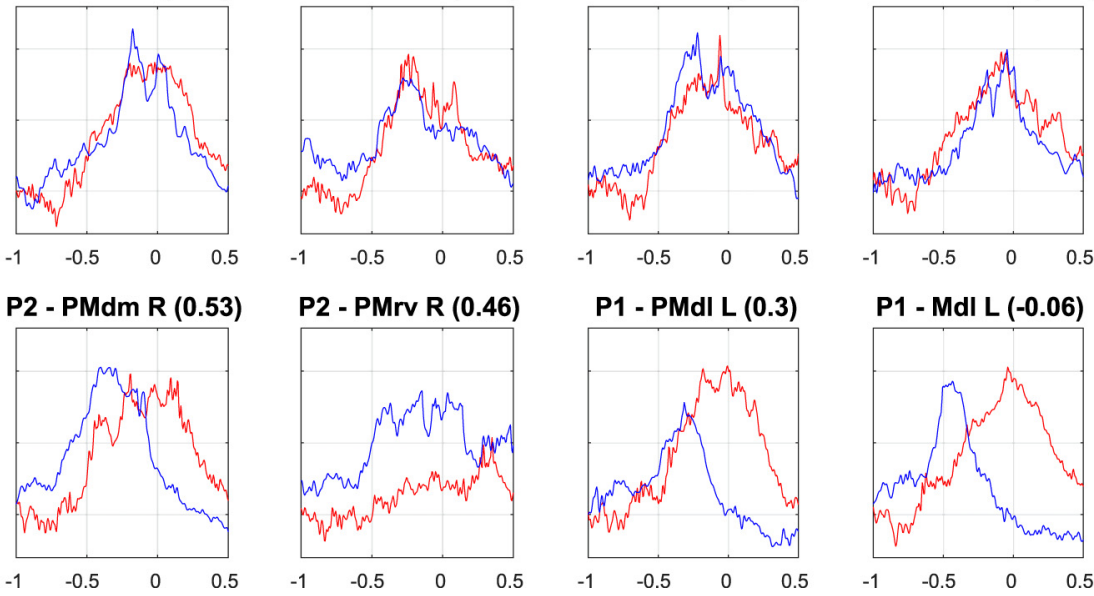

Figure 10. SEEG validation of HGA modulations. Comparison between SEEG single-subjects $t$-value for HGA (blue) and group-level MEG results (red curves). Subject number, brain areas, and the correlation coefficient between the curves are indicated on the top of each panel.

analyses showed that visuomotor mapping resides in three distinct and partly overlapping subnetworks with time-evolving cortico-cortical and cortico-subcortical interactions. Approximately $0.5 \mathrm{~s}$ before finger movement, visual and parietal regions coordinate with sensorimotor and premotor areas ( $\mathrm{LCl}$ in Fig. $5 B$; blue curve in Fig. 5A). Subsequently, the sensorimotor regions, the dorsal frontoparietal circuit, the medial prefrontal regions, the basal ganglia, and the thalamus (LC2 in Fig. $5 \mathrm{C}$ ) dominated the FC pattern. The dorsal frontoparietal circuit, known to support visuomotor transformations and goal-directed attentional processes (Wise et al., 1996; Wise and Murray, 2000; Corbetta and Shulman, 2002; Culham and Valyear, 2006), is tightly coupled with the sensorimotor and associative frontostriatal circuit for a brief period around $0.35 \mathrm{~s}$ before action (red curve in Fig. 5A). This FC network includes medial prefrontal areas, such as the dorsomedial prefrontal cortex (PFCdm), midcingulate cortex (MCC), anterior cingulate cortex (ACC) and rostro-medial prefrontal (PFrm), with a strongest increase in HGA in the MCC and PFCdm (Fig. 2). The involvement of medial prefrontal areas may correspond to the activation of visuomotor-related neural populations of the rostral cingulate zone (RCZ), a key node of the human motor system (Picard and Strick, 1996; Amiez and Petrides, 2014). At the subcortical level, the dorsal striatum (caudate nucleus and putamen), globus pallidus and thalamus displayed the strongest HGA in the hemisphere contralateral to the motor response (Fig. 2). Indeed, intracranial recordings from patients with motor disorders have described HGA in the subthalamic nucleus (Amirnovin et al., 2004; Alegre et al., 2005; Androulidakis et al., 2007; Lalo et al., 2008), globus pallidus (Tsang et al., 2012) and thalamus (Brücke et al., 2013) during different types of motor behaviors. Highgamma oscillatory activity in the subthalamic nucleus (STN) and globus pallidus (GPi) has been found to be coherent with cortical activity during voluntary movement (Cassidy et al., 2002; Brown, 2003). Granger causality analysis also showed that STN drives activity in M1 (Litvak et al., 2012), thus suggesting that HGA in motor areas is due to propagating activity from the basal ganglia through the thalamus (Brücke et al., 2013). Our results showed that the caudate nucleus and thalamus are coupled with the sensorimotor cortex and the dorsal frontoparietal networks (LC2 in Fig. $5 C$ ), thus confirming a dynamic coordination between cortical and subcortical regions also during visuomotor behaviors most prominently during the planning phase before movement initiation.

Several lines of evidence suggest that the reported subcortical activations are primarily local, rather than due to leakage from cortical areas. First, we observed that the putamen significantly activated only in the left hemisphere, whereas the thalamus was activated bilaterally (Fig. 2). If the bilateral activation in the thalamus were due to leakage from cortical areas, we would have expected a bilateral activation also in the putamen, given that the thalamus is deeper than the putamen. Second, no significant correlation was found between the SFL and distance for the thalamus and caudate nucleus (Fig. 8). Third, the average dipole orientation for the thalamus and caudate nucleus are significantly different (i.e., the normalized inner product is $<1$ ) and the distribution of values covers a wide range from -0.45 to $\sim 0.85$. This suggests that the thalamus and caudate nucleus lack a systematic similarity in dipole orientation (Fig. 9A). Fourth, the mean Pearson correlation values and the corresponding coefficient of determination do not saturate at high values and show a modulation similar to the average time course of FCD (Fig. 9B). This suggests a lack of strong covariance, as would be expected if leakage effects were dominating.

Nevertheless, we cannot a priori exclude that the observed subcortical increases in HGA and FC patterns may be due to complex configurations of cortical activations. Indeed, we suggest that HGA estimation at subcortical areas and FC analysis should not be performed blindly. Rather, the analysis of raw correlations and dipole orientations provide important insight into the origin of the results. Experimentally, simultaneous MEG and subcortical measures of HGA from intracranial recordings would be required to confirm or disprove the ability of MEG to capture subcortical HGA and FC.

Overall, our result suggests that the basal ganglia form a dynamic functional network, which may allow the coordination within and across different processing streams in the basal ganglia (Brown, 2003) and facilitate motor output (Cheyne and Ferrari, 2013). The involvement of sensorimotor and associative frontostriatal circuits, classically thought to be involved in habits (Yin and Knowlton, 2006; Graybiel, 2008; Ashby et al., 2010), also suggests that performance of arbitrary visuomotor mappings can be viewed as a form of acquainted instrumental behavior (Brovelli et al., 2015), the gradual consolidation of which would lead to 
the formation of habitual responses (Dickinson, 1985; Dickinson and Balleine, 1993). We therefore suggest that the FCD pattern observed for LC2 depicts how motor plans circulate in the sensorimotor and associative cortico-striatal loop in coordination with frontoparietal circuits.

Finally, a last subnetwork (LC3 in Fig. 5D) primarily emerging after motor response peaking around $0.2 \mathrm{~s}$ (green curve in Fig. $5 \mathrm{~A}$ ) that is involved in the bilateral sensorimotor regions, the left frontoparietal network, and visual areas. The involvement of bilateral sensorimotor regions indicates that this network mediates cortico-cortical interhemispheric coordination processes via the corpus callosum. Such interhemispheric coupling may support the selective inhibition of inappropriate responses occurring between motor and premotor cortices across hemispheres in situations when multiple choices are available (Duque et al., 2013; Burle et al., 2016). Alternatively, this network may be involved in the processing of the somatosensory reafference at the end of the movement and/or the processing action's outcome.

To exclude potential confounds due to volume conduction or limited spatial resolution of MEG and beam-forming technique (Bastos and Schoffelen, 2015), we showed that local field potentials derived from SEEG recordings displayed a significant increase in HGA in these regions (Fig. 10, Table 2). We confirmed invasively that brain regions of the dorsal frontoparietal network and the sensorimotor and/or associative frontostriatal circuits display local increase in HGA, which do not result from volume conduction effects.

\section{Toward a better understanding of cognitive architectures}

We computed the density of the thresholded FC and FCD graphs as a function of significance threshold (Fig. 4) using the FDRcontrolling procedure to estimate the relevant range of $q$-values. For $q<0.05$, the density of the visuomotor-related FC network is $<10 \%$ ( $\sim 5 \%$ for $q<0.05)$. This shows that visuomotor-related FC is not dense, but sparse. Future work on the relation between structural and FC may provide clues of how anatomical connectivity between brain areas shapes how neural information flows and constrains the dynamics of FC patterns.

A second characteristic of FC is its nonstationarity nature. Indeed, fMRI investigations have shown that resting-state networks display nonstationarity and evolve dynamically over tens of seconds to minutes (Hutchison et al., 2013; Yeo et al., 2014; Allen et al., 2014; Calhoun et al., 2014; Cole et al., 2014; Zalesky et al., 2014; Hansen et al., 2015). Interareal phase synchronization is known to display nonstationarity and to underlie perception and executive functions (Pesaran et al., 2008; Hipp et al., 2011; Salazar et al., 2012). Our results further support the notion that FC is nonstationary and evolves over timescales relevant for visuomotor integration in the order of tens to hundreds of milliseconds (Figs. 3B, 5). Modeling studies of resting-state activity suggest that nonstationarity arises from the out-of-equilibrium sampling of alternative dynamical modes (Deco and Jirsa, 2012; Deco et al., 2015; Hansen et al., 2015). Switching between collective dynamical states has the potential to induce network-wide reorganization of information sharing and routing patterns and thus provides an effective mechanism for flexible interareal communication (Battaglia et al., 2012; Kirst et al., 2016). We suggest that such underlying mechanisms mediating spontaneous large-scale dynamics may also underlie task-related activity at shorter timescale. In addition, our results may provide the basis for linking similar patterns of FCD that have been observed, albeit over different timescales, during visuomotor learning (Bassett et al., 2011; Heitger et al., 2012).
A third characteristic of FCD is the presence of multiple and spatially overlapping subnetworks. Each brain area can participate in multiple subnetworks depending of task demands. In analogy to the concept of cell assembly, where single neurons can participate in multiple functional populations depending on context, a subnetwork may be viewed as a brain assembly. A brain assembly would constitute a dynamic entity, the constituents of which engage in multiple subnetworks in a time-dependent manner (Fig. 6). Spatially, areal flexibility was larger for associative parietal regions such as the SPC, PCm, and PPC, together with the dorsal premotor cortex, which represent the core cortical network for visuomotor transformation (Fig. 7B). Temporally, network flexibility showed a maximum around $0.4 \mathrm{~s}$ before finger movement, which corresponds to the moment when the three subnetworks interact more strongly (Fig. 7A). We therefore suggest that visuomotor integration processes occur at this time interval, when the level of interactions between multiple brain assemblies is strongest.

To conclude, our study confirms that executive functions arise from the dynamic coordination of neural activity over largescale networks (Varela et al., 2001; Bressler and Menon, 2010; von der Malsburg et al., 2010). More precisely, the results supports the notion that functional specialization is due to the interplay of multiple and spatially overlapping subnetworks, rather than properties of single brain regions (Fedorenko and ThompsonSchill, 2014; Petersen and Sporns, 2015). Future work investigating how subnetworks participate differently depending on task demands may provide a better understanding of the cognitive architectures of executive functions (Dehaene et al., 2015).

\section{References}

Ahn YY, Bagrow JP, Lehmann S (2010) Link communities reveal multiscale complexity in networks. Nature 466:761-764. CrossRef Medline

Alegre M, Alonso-Frech F, Rodríguez-Oroz MC, Guridi J, Zamarbide I, Valencia M, Manrique M, Obeso JA, Artieda J (2005) Movement-related changes in oscillatory activity in the human subthalamic nucleus: ipsilateral vs. contralateral movements. Eur J Neurosci 22:2315-2324. Medline

Allen EA, Damaraju E, Plis SM, Erhardt EB, Eichele T, Calhoun VD (2014) Tracking whole-brain connectivity dynamics in the resting state. Cereb Cortex 24:663-676. CrossRef Medline

Amiez C, Petrides M (2014) Neuroimaging evidence of the anatomofunctional organization of the human cingulate motor areas. Cereb Cortex 24:563-578. CrossRef Medline

Amirnovin R, Williams ZM, Cosgrove GR, Eskandar EN (2004) Visually guided movements suppress subthalamic oscillations in Parkinson's disease patients. J Neurosci 24:11302-11306. CrossRef Medline

Androulidakis AG, Kühn AA, Chen CC, Blomstedt P, Kempf F, Kupsch A, Kupsch A, Schneider GH, Doyle L, Dowsey-Limousin P, Hariz MI, Brown P (2007) Dopaminergic therapy promotes lateralized motor activity in the subthalamic area in Parkinson's disease. Brain 130:457-468. CrossRef Medline

Ashby FG, Turner BO, Horvitz JC (2010 May) (2010) Cortical and basal ganglia contributions to habit learning and automaticity. Trends Cogn Sci 14:208-215. CrossRef Medline

Auzias G, Lefèvre J, Le Troter A, Fischer C, Perrot M, Régis J, Coulon O (2013) Model-driven harmonic parameterization of the cortical surface: HIP-HOP. IEEE Trans Med Imaging 32:873-887. CrossRef Medline

Auzias G, Coulon O, Brovelli A (2016) MarsAtlas: A cortical parcellation atlas for functional mapping. Hum Brain Mapp 37:1573-1592. CrossRef Medline

Ball T, Demandt E, Mutschler I, Neitzel E, Mehring C, Vogt K, Aertsen A, Schulze-Bonhage A (2008) Movement related activity in the high gamma range of the human EEG. Neuroimage 41:302-310. CrossRef Medline

Bassett DS, Wymbs NF, Porter MA, Mucha PJ, Carlson JM, Grafton ST (2011) Dynamic reconfiguration of human brain networks during learning. Proc Natl Acad Sci U S A 108:7641-7646. CrossRef Medline

Bassett DS, Porter MA, Wymbs NF, Grafton ST, Carlson JM, Mucha PJ 
(2013) Robust detection of dynamic community structure in networks. Chaos 23:013142. CrossRef Medline

Bassett DS, Wymbs NF, Porter MA, Mucha PJ, Grafton ST (2014) Crosslinked structure of network evolution. Chaos 24:013112. CrossRef Medline

Bastos AM, Schoffelen JM (2015) A tutorial review of functional connectivity analysis methods and their interpretational pitfalls. Front Syst Neurosci 9:175. CrossRef Medline

Battaglia D, Witt A, Wolf F, Geisel T (2012) Dynamic effective connectivity of inter-areal brain circuits. PLoS Comp Biol 8:e1002438. CrossRef Medline

Benjamini Y, Yosef H (1995) Controlling the false discovery rate: a practical and powerful approach to multiple testing. Journal of the Royal Statistical Society Series B (Methodological) 57:289-3001.

Blondel VD, Guillaume JL, Lambiotte R, Lefebvre E (2008) Fast unfolding of communities in large networks. Journal of Statistical Mechanics: Theory and Experiment 10:P1000.

Braun U, Schäfer A, Walter H, Erk S, Romanczuk-Seiferth N, Haddad L, Schweiger JI, Grimm O, Heinz A, Tost H, Meyer-Lindenberg A, Bassett DS (2015) Dynamic reconfiguration of frontal brain networks during executive cognition in humans. Proc Natl Acad Sci U S A 112:1167811683. CrossRef Medline

Bressler SL, Menon V (2010) Large-scale brain networks in cognition: emerging methods and principles. Trends Cogn Sci 14:277-290. CrossRef Medline

Brovelli A, Lachaux JP, Kahane P, Boussaoud D (2005) High gamma frequency oscillatory activity dissociates attention from intention in the human premotor cortex. Neuroimage 28:154-164. CrossRef Medline

Brovelli A, Chicharro D, Badier JM, Wang H, Jirsa V (2015) Characterization of cortical networks and cortico-cortical functional connectivity mediating arbitrary visuomotor mapping. J Neurosci 35:12643-12658. CrossRef Medline

Brown P (2003) Oscillatory nature of human basal ganglia activity: relationship to the pathophysiology of Parkinson's disease. Mov Disord 18:357363. CrossRef Medline

Brücke C, Bock A, Huebl J, Krauss JK, Schönecker T, Schneider GH, Brown P, Kühn AA (2013) Thalamic gamma oscillations correlate with reaction time in a Go/noGo task in patients with essential tremor. Neuroimage 75:36-45. CrossRef Medline

Burle B, van den Wildenberg WP, Spieser L, Ridderinkhof KR (2016) Preventing (impulsive) errors: Electrophysiological evidence for online inhibitory control over incorrect responses. Psychophysiology 53:10081019. CrossRef Medline

Buzsáki G, Schomburg EW (2015) What does gamma coherence tell us about inter-regional neural communication? Nat Neurosci 18:484-489. CrossRef Medline

Cachia A, Mangin JF, Rivière D, Papadopoulos-Orfanos D, Kherif F, Bloch I, Régis J (2003) A generic framework for the parcellation of the cortical surface into gyri using geodesic Voronoï diagrams. Medical Image Analysis 7:403-416. CrossRef Medline

Calhoun VD, Miller R, Pearlson G, Adalı T (2014) The chronnectome: time-varying connectivity networks as the next frontier in fMRI data discovery. Neuron 84:262-274. CrossRef Medline

Cassidy M, Mazzone P, Oliviero A, Insola A, Tonali P, Di Lazzaro V, Brown P (2002) Movement-related changes in synchronization in the human basal ganglia. Brain. 125:1235-1246. CrossRef Medline

Cheyne D, Ferrari P (2013) MEG studies of motor cortex gamma oscillations: evidence for a gamma "fingerprint" in the brain? Front Hum Neurosci 7:575. CrossRef Medline

Cole MW, Bassett DS, Power JD, Braver TS, Petersen SE (2014) Intrinsic and task-evoked network architectures of the human brain. Neuron 83: 238-251. CrossRef Medline

Corbetta M, Shulman GL (2002) Control of goal-directed and stimulusdriven attention in the brain. Nat Rev Neurosci 3:201-215. Medline

Coupé P, Yger P, Prima S, Hellier P, Kervrann C, Barillot C (2008 Apr) (2008) An optimized blockwise nonlocal means denoising filter for 3-D magnetic resonance images. IEEE Trans Med Imaging 27:425-441. CrossRef Medline

Crone NE, Sinai A, Korzeniewska A (2006) High-frequency gamma oscillations and human brain mapping with electrocorticography. Prog Brain Res 159:275-295. CrossRef Medline
Culham JC, Valyear KF (2006) Human parietal cortex in action. Curr Opin Neurobiol 16:205-212. CrossRef Medline

Darvas F, Scherer R, Ojemann JG, Rao RP, Miller KJ, Sorensen LB (2010) High gamma mapping using EEG. Neuroimage 49:930-938. CrossRef Medline

Davison EN, Schlesinger KJ, Bassett DS, Lynall ME, Miller MB, Grafton ST, Carlson JM. Brain network adaptability across task states (2015) PLoS Comput Biol 8;11:e1004029.

Deco G, Jirsa VK (2012) Ongoing cortical activity at rest: criticality, multistability, and ghost attractors. J Neurosci 32:3366-3375. CrossRef Medline

Deco G, Tononi G, Boly M, Kringelbach ML (2015) Rethinking segregation and integration: contributions of whole-brain modelling. Nat Rev Neurosci 16:430-439. CrossRef Medline

Dehaene S, Dudai Y, Konen C (2015) Cognitive architectures. Neuron 88:1. CrossRef Medline

Desikan RS, Ségonne F, Fischl B, Quinn BT, Dickerson BC, Blacker D, Buckner RL, Dale AM, Maguire RP, Hyman BT, Albert MS, Killiany RJ (2006) An automated labeling system for subdividing the human cerebral cortex on MRI scans into gyral based regions of interest. Neuroimage 31:968980. CrossRef Medline

Dickinson A, Balleine B (1993) Actions and responses: the dual psychology of behaviour. In: Spatial representation: problems in philosophy and psychology (Eilan N, McCarthy RA, Brewer B, eds), pp 277-293. Malden, MA: Blackwell Publishers.

Dickinson AD (1985) Actions and habits: the development of behavioral autonomy. Philos Trans R Soc Lond B Biol Sci 308:67-78. CrossRef

Duque J, Olivier E, Rushworth M (2013) Top-down inhibitory control exerted by the medial frontal cortex during action selection under conflict. J Cogn Neurosci 25:1634-1648. CrossRef Medline

Fedorenko E, Thompson-Schill SL (2014) Reworking the language network. Trends Cogn Sci 18:120-126. CrossRef Medline

Fischl B, Salat DH, Busa E, Albert M, Dieterich M, Haselgrove C, van der Kouwe A, Killiany R, Kennedy D, Klaveness S, Montillo A, Makris N, Rosen B, Dale AM (2002) Whole brain segmentation: automated labeling of neuroanatomical structures in the human brain. Neuron 33:341355. CrossRef Medline

Fries P (2015) Rhythms for cognition: communication through coherence. Neuron 88:220-235. CrossRef Medline

Goodman SN (1999a) Toward evidence-based medical statistics. 1: The P value fallacy. Ann Int Med 130:995-1004. CrossRef Medline

Goodman SN (1999b) Toward evidence-based medical statistics. 2: the Bayes factor. Ann Int Med 130:1005-1013. CrossRef Medline

Goodman SN (2001) Of P-values and Bayes: a modest proposal. Epidemiology 12:295-297. CrossRef Medline

Graybiel AM (2008) Habits, rituals and the evaluative brain. Annu Rev Neurosci 31:359-387. CrossRef Medline

Gross J, Kujala J, Hamalainen M, Timmermann L, Schnitzler A, Salmelin R (2001) Dynamic imaging of coherent sources: studying neural interactions in the human brain. Proc Natl Acad Sci U S A 98:694-699. CrossRef Medline

Hadj-Bouziane F, Meunier M, Boussaoud D (2003) Conditional visuomotor learning in primates: a key role for the basal ganglia. J Physiol Paris 97:567-579. CrossRef Medline

Hansen EC, Battaglia D, Spiegler A, Deco G, Jirsa VK (2015) Functional connectivity dynamics: modeling the switching behavior of the resting state. Neuroimage 105:525-535. CrossRef Medline

Heitger MH, Ronsse R, Dhollander T, Dupont P, Caeyenberghs K, Swinnen SP (2012) Motor learning-induced changes in functional brain connectivity as revealed by means of graph-theoretical network analysis. Neuroimage 61:633-650. CrossRef Medline

Hermes D, Miller KJ, Noordmans HJ, Vansteensel MJ, Ramsey NF (2010) Automated electrocorticographic electrode localization on individually rendered brain surfaces. J Neurosci Methods 185:293-298. CrossRef Medline

Hipp JF, Engel AK, Siegel M (2011) Oscillatory synchronization in largescale cortical networks predicts perception. Neuron 69:387-396. CrossRef Medline

Hutchison RM, Womelsdorf T, Allen EA, Bandettini PA, Calhoun VD, Corbetta M, Della Penna S, Duyn JH, Glover GH, Gonzalez-Castillo J, Handwerker DA, Keilholz S, Kiviniemi V, Leopold DA, de Pasquale F, Sporns O, Walter M, Chang C (2013) Dynamic functional connectivity: 
promise, issues, and interpretations. Neuroimage 80:360-378. CrossRef Medline

Jerbi K, Ossandón T, Hamamé CM, Senova S, Dalal SS, Jung J, Minotti L, Bertrand O, Berthoz A, Kahane P, Lachaux JP (2009) Task-related gamma-band dynamics from an intracerebral perspective: review and implications for surface EEG and MEG. Hum Brain Mapp 30:1758-1771. CrossRef Medline

Kirst C, Timme M, Battaglia D (2016) Dynamic information routing in complex networks. Nat Commun 7:11061. CrossRef Medline

Ko AL, Weaver KE, Hakimian S, Ojemann JG (2013) Identifying functional networks using endogenous connectivity in gamma band electrocorticography. Brain Connect 3:491-502. CrossRef Medline

Lachaux JP, Axmacher N, Mormann F, Halgren E, Crone NE (2012) Highfrequency neural activity and human cognition: past, present and possible future of intracranial EEG research. Prog Neurobiol 98:279-301. CrossRef Medline

Lalo E, Thobois S, Sharott A, Polo G, Mertens P, Pogosyan A, Brown P (2008) Patterns of bidirectional communication between cortex and basal ganglia during movement in patients with Parkinson disease. J Neurosci 28: 3008-3016. CrossRef Medline

Lancaster JL, Woldorff MG, Parsons LM, Liotti M, Freitas CS, Rainey L, Kochunov PV, Nickerson D, Mikiten SA, Fox PT (2000) Automated Talairach atlas labels for functional brain mapping. Hum Brain Mapp 10:120-131. CrossRef Medline

Litvak V, Eusebio A, Jha A, Oostenveld R, Barnes G, Foltynie T, Limousin P, Zrinzo L, Hariz MI, Friston K, Brown P (2012) Movement-related changes in local and long-range synchronization in Parkinson's disease revealed by simultaneous MEG and intracranial recordings. J Neurosci 32:10541-10553. CrossRef Medline

Mangin JF, Rivière D, Cachia A, Duchesnay E, Cointepas Y, PapadopoulosOrfanos D, Scifo P, Ochiai T, Brunelle F, Régis J (2004) A framework to study the cortical folding patterns. Neuroimage. 23:S129-S138. CrossRef Medline

Mitra PP, Pesaran B (1999) Analysis of dynamic brain imaging data. Biophys J 76:691-708. CrossRef Medline

Murray EA, Bussey TJ, Wise SP (2000) Role of prefrontal cortex in a network for arbitrary visuomotor mapping. Exp Brain Res 133:114-129. CrossRef Medline

Nolte G (2003) The magnetic lead field theorem in the quasi-static approximation and its use for MEG forward calculation in realistic volume conductors. Phys Med Biol 48:3637-3652. CrossRef Medline

Pandya DN, Yeterian EH (1985) Architecture and connections of cortical association areas. Cereb Cortex 4:3-61. CrossRef

Passingham RE, Toni I, Rushworth MF (2000) Specialisation within the prefrontal cortex: the ventral prefrontal cortex and associative learning. Exp Brain Res 133:103-113. CrossRef Medline

Percival DB, Walden AT (1993) Spectral analysis for physical applications. Cambridge: Cambridge University.

Perrot M, Rivière D, Mangin JF (2011) Cortical sulci recognition and spatial normalization. Med Image Anal 15:529-550. CrossRef Medline

Pesaran B, Nelson MJ, Andersen RA (2008) Free choice activates a decision circuit between frontal and parietal cortex. Nature 453:406-409. CrossRef Medline

Petersen SE, Sporns O (2015) Brain networks and cognitive architectures. Neuron 88:207-219. CrossRef Medline

Petrides M (2005) Lateral prefrontal cortex: architectonic and functional organization. Philos Trans R Soc Lond B Biol Sci 360:781-795. CrossRef Medline

Picard N, Strick PL (1996) Motor areas of the medial wall: a review of their location and functional activation. Cereb Cortex 6:342-353. CrossRef Medline

Ray S, Maunsell JH (2011) Different origins of gamma rhythm and high- gamma activity in macaque visual cortex. PLoS Biol 9:e1000610. CrossRef Medline

Ray S, Crone NE, Niebur E, Franaszczuk PJ, Hsiao SS (2008) Neural correlates of high-gamma oscillations $(60-200 \mathrm{~Hz})$ in macaque local field potentials and their potential implications in electrocorticography. J Neurosci 28:11526-11536. CrossRef Medline

Régis J, Mangin JF, Ochiai T, Frouin V, Riviére D, Cachia A, Tamura M, Samson Y (2005) "Sulcal root" generic model: a hypothesis to overcome the variability of the human cortex folding patterns. Neurologia Medico Chirurgica 45:1-17. CrossRef Medline

Rubinov M, Sporns O (2010) Complex network measures of brain connectivity: uses and interpretations. Neuroimage 52:1059-1069. CrossRef Medline

Salazar RF, Dotson NM, Bressler SL, Gray CM (2012) Content-specific fronto-parietal synchronization during visual working memory. Science 338:1097-1100. CrossRef Medline

Smith AC, Frank LM, Wirth S, Yanike M, Hu D, Kubota Y, Graybiel AM, Suzuki WA, Brown EN (2004) Dynamic analysis of learning in behavioral experiments. J Neurosci 24:447-461. CrossRef Medline

Stephens M, Balding DJ (2009) Bayesian statistical methods for genetic associationstudies. Nat Rev Genet 10:681-690. CrossRef Medline

Tou JT, Gonzalez RC (1974) Pattern recognition principles. Reading, MA: Addison-Wesley.

Traud AL, Kelsic ED, Mucha PJ, Porter MA (2011) Comparing community structure to characteristics in online collegiate social networks. SIAM Review 53:526-543. CrossRef

Tsang EW, Hamani C, Moro E, Mazzella F, Lozano AM, Hodaie M, Yeh IJ, Chen R (2012) Movement related potentials and oscillatory activities in the human internal globus pallidus during voluntary movements. J Neurol Neurosurg Psychiatry 83:91-97. CrossRef Medline

Tzourio-Mazoyer N, Landeau B, Papathanassiou D, Crivello F, Etard O, Delcroix N, Mazoyer B, Joliot M (2002) Automated anatomical labeling of activations in SPM using a macroscopic anatomical parcellation of the MNI MRI single-subject brain. Neuroimage 15:273-289. CrossRef Medline

Van Essen DC, Drury HA (1997) Structural and functional analyses of human cerebral cortex using a surface-based atlas. J Neurosci 17:7079-7102. Medline

Varela F, Lachaux JP, Rodriguez E, Martinerie J (2001) The brainweb: phase synchronization and large-scale integration. Nat Rev Neurosci 2:229 239. CrossRef Medline

Van Veen BD, van Drongelen W, Yuchtman M, Suzuki A (1997) Localization of brain electrical activity via linearly constrained minimum variance spatial filtering. IEEE Trans Biomed Eng 44:867-880. CrossRef Medline

Vidal JR, Chaumon M, O'Regan JK, Tallon-Baudry C (2006) Visual grouping and the focusing of attention induce gamma-band oscillations at different frequencies in human magnetoencephalogram signals. J Cogn Neurosci 18:1850-1862. CrossRef Medline

Von der Malsburg C, Phillips PEM, Singer W (2010) Dynamic coordination in the brain. Cambridge, MA: MIT.

Wise SP, Murray EA (2000) Arbitrary associations between antecedents and actions. Trends Neurosci 23:271-276. CrossRef Medline

Wise SP, di Pellegrino G, Boussaoud D (1996) The premotor cortex and nonstandard sensorimotor mapping. Can J Physiol Pharmacol 74:469_ 482. Medline

Yeo BT, Krienen FM, Chee MW, Buckner RL (2014) Estimates of segregation and overlap of functional connectivity networks in the human cerebral cortex. Neuroimage 88:212-227. CrossRef Medline

Yin HH, Knowlton BJ (2006) The role of the basal ganglia in habit formation. Nat Rev Neurosci 7:464-476. CrossRef Medline

Zalesky A, Fornito A, Cocchi L, Gollo LL, Breakspear M (2014) Timeresolved resting-state brain networks. Proc Natl Acad Sci U S A 111: 10341-10346. CrossRef Medline 